\title{
Characterisation and Manipulation of Proton Beams Accelerated by Ultra-Short and High-Contrast Laser Pulses
}

\author{
Sargis Ter-Avetisyan ${ }^{1}$, Mathias Schnürer ${ }^{2}$ and Peter V Nickles 2,3 \\ 'School of Mathematics and Physics, The Queen's University of Belfast, \\ ${ }_{2}^{2}$ Nonlinear optics and short pulse spectroscopy, Max Born Institute, Berlin, \\ ${ }^{3}$ Gwangju Institute of Science and Technology, WCU Department, \\ ${ }^{1} U K$ \\ ${ }^{2}$ Germany \\ ${ }^{3}$ Rep. of Korea
}

\section{Introduction}

Laser-driven acceleration of particle beams is a burgeoning field of research, based on the possibility of creating ultra-large electric fields in plasma, largely exceeding current limits of conventional acceleration technology. Research in this area has led to very significant progression regarding the acceleration of electrons and ions. Laser-driven ion sources have unique properties: high brightness (about $10^{13}$ protons/ions per shot), high current (in kiloAmpere range), ultra-low emittance, and short pulse duration (less than 1 ps), opening prospects for a broad range of applications. Recent advances in the laser technology have led to even further enhancements in the provision of extremely short and high-intensity pulses, which can further improve the accelerated ion beam specifications particularly regarding maximum ion energy and ion flux which are demanding most of the potential applications. These developments have stimulated an emergence of advanced diagnostics for measuring complex plasma effects.

The absorption mechanisms of laser radiation at the target are the basic processes, which indeed defines the whole ion acceleration scenario. For laser intensities, where the classical normalized momentum of electrons quivering in the laser electric field: $a=8.53 \times 10^{-10}(\mu \mathrm{m})$ $I^{1 / 2}\left(\mathrm{~W} / \mathrm{cm}^{2}\right)>1$, the electrons become relativistic and the effect of the laser magnetic field is no longer negligible. The perpendicular component of the Lorentz force $e \mathbf{v} \times \mathbf{B}$ couples with the electric force to drive the electrons in the laser propagation direction (Wilks et al., 1992, Lefebvre \& Bonnaud, 1997, Kruer \& Estabrook, 1985) in contrast to inverse bremsstrahlung and resonance absorption, which causes the quiver motion of the electrons in the laser field. The ponderomotive force drives the electrons with a step- or plateau-like density profile and has a strong directionality along the laser propagation direction. Electron temperatures about $1 \mathrm{MeV}$ have been measured (Malka \& Miquel, 1996). The laser energy transfer to the hot electrons can also be out carried by fast plasma waves through the nonlinear ponderomotive force (Tajima \& Dawson, 1979) and by laser field itself (Pukhov et al., 1999). 
Whereas the ions from the target front will be accelerated normal to the target front surface in the ambipolar expansion of the plasma, the hot electron component created directly by the laser pulse in the plasma plume will propagate through the target. It has typically a divergence between $5^{\circ}-50^{\circ}$, density of the order of the critical density $\left(10^{20}-10^{21} \mathrm{~cm}^{-3}\right)$ and a temperature of the order of the laser ponderomotive potential.

The free motion of this hot electron beam through the target requires a return current that locally compensates the flow of the hot electrons (Passoni et al., 2004). It will be provided by the target material (for metallic target - conduction electrons, insulators - the background free electron population created by field and thermal ionization). Since the density of the background electron population in both cases is of the order of the solid density, much bigger than the fast electron density, the required velocity for current neutralization is small and their temperature is much lower than that of the hot electrons (Tikhonchuk, 2002). Finally the physical parameters and dynamics of these two electron populations will define the electrostatic sheath field which is created at the vacuum - solid interface and accelerates ions to high energies.

The ions are created and accelerated either at the target rear surface (Snavely et al., 2000, Mackinnon et al., 2001, Hegelich et al., 2002) through the self-consistent electrostatic accelerating field generated by fast electrons escaping in vacuum (so-called target normal sheath acceleration-TNSA mechanism) or at the target front surface, illuminated by the laser (Clark et al., 2000a, Maksimchuk et al., 2000, Clark et al., 2000b). Particle-in-cell (PIC) simulations suggest a variety of mechanisms that may be responsible for acceleration at the front surface: formation of multiple collisionless electrostatic shocks (Denavit, 1992, Silva et al., 2004, Wei et al., 2004); a solitary wave produced by shock-wave decay in a plasma slab (Zhidkov et al., 2002); or a mechanism wherein the ponderomotive pressure of the short laser pulse displaces the background electrons, and the ions are accelerated by the electrostatic field of the propagating double layer (Shorokhov \& Pukhov, 2004). However, all these mechanisms are relying on ion acceleration in the electrostatic field created due to charge displacement driven by the laser field.

These scenarios are not mutually exclusive. Their relative contributions depend strongly upon the particular target and laser parameters and can contribute to the generation of electrons and, in turn, to ion acceleration mechanisms. Particle-in-cell (PIC) simulations by Wilks et al., (2001), and Pukhov, (2001), and observations by Zepf et al., (2003), and Karsch et al., (2003), show that ions can be produced at the target front and the rear sides simultaneously, even if the generation processes are quite different. Here the laser pulse contrast has a profound effect on accelerated ions and their cut-off energy (Mackinnon et al., 2002, Kaluza et al., 2004, Lindau et al., 2005).

The acceleration is most effective on light ions (specifically protons), which are usually present on target surfaces in the form of contaminants like hydrocarbons and water, or can be present among the constituents of the solid target (e.g. as in plastic targets). The heaviest ion population of the target provides a positive charge, which offers much more inertia and makes the charge separation responsible for the huge accelerating field. Part of this heavy population can also be effectively accelerated, on a longer time scale, if the protons are not enough to acquire most of the energy contained in the electric field, or if protons are removed before the arrival of the laser pulse.

The complex, non-linear nature of laser-driven plasma dynamics and ion acceleration phenomena requires the development of an innovative diagnostic complex allowing 
simultaneous measurements of different plasma parameters (using visible- and XUV-light, X-rays, Gamma-rays, ion and electrons) with high temporal, spectral and spatial resolution together with laser pulse parameters. This requirement is especially important because of both: shot-to-shot fluctuations of laser pulse parameters and inherent shot-to-shot variations in the local target parameters, which can derogate the whole plasma dynamics.

In laser-matter interaction studies one of the important research tasks is to investigate the energy distributions of emerging charged particles, in which the different laser energy absorption mechanisms are hidden and the energy redistribution among the plasma components become apparent. As a basic diagnostic the Thomson spectrometer has been widely and successfully used for analyzing the energy spectra of laser accelerated charged particles. In Fig.1 a typical experimental setup of laser plasma interaction experiments with Thomson parabola spectrometers and, as an example, spectral traces recorded with absolute calibrated micro-channel-plate (MCP) detector (Ter-Avetisyan, et al., 2005) are shown.
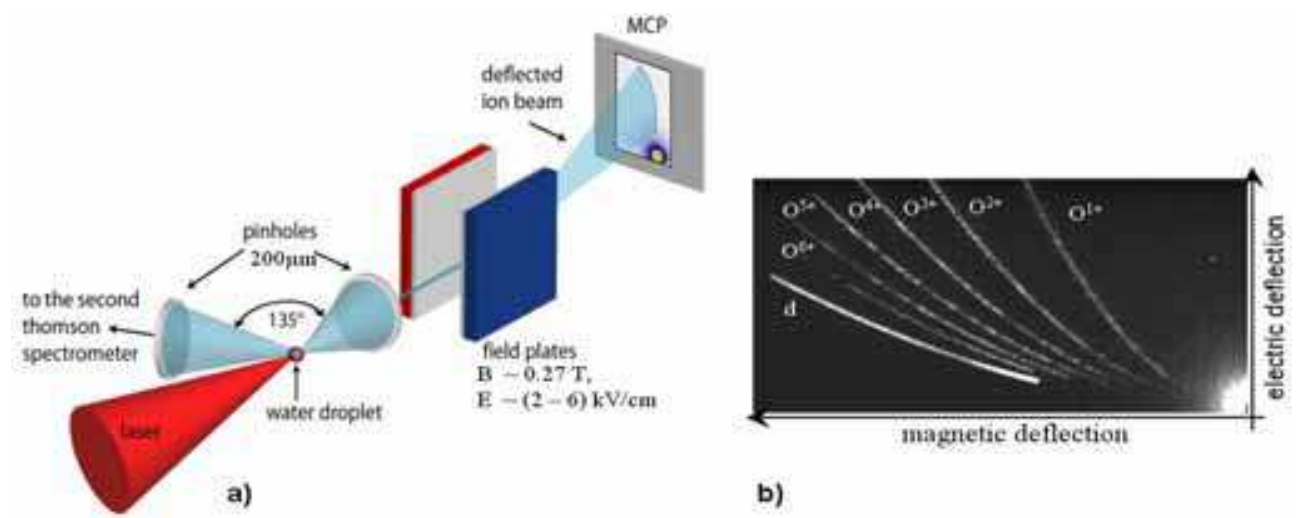

b)

Fig. 1. a) A typical experimental setup of laser plasma interaction experiments with Thomson parabola spectrometers and, as an example, b) measured accelerated ion spectra from heavy water droplet irradiated at $35 \mathrm{fs}, \sim 1 \mathrm{~J}, \sim 10^{19} \mathrm{~W} / \mathrm{cm}^{2}$ intensity is shown.

Several modifications of the basic spectrometer design allow a comprehensive and precise analysis of ion acceleration. In particular, (i) the simultaneous measurement of ion and electron spectra in the same direction allow to understand the measured particularities in the ion spectra resulting from the evolution of a two-electron component (hot and cold) plasma (Ter-Avetisyan et al., 2004a). (ii) The complexity of the temporal (Ter-Avetisyan, et al., 2005) and spatial characteristics (Schreiber et al., 2006) of laser driven ion source could be demonstrated by precise measurement of the proton/ion trajectories, and its applicability for proton deflectometry (Ter-Avetisyan et al., 2008, Sokollik et al., 2008), and (iii) proton source tomography revealed detailed properties of the laser driven ion source (TerAvetisyan et al., 2009a). Latest is crucial in view of planning proton beam steering systems. The comprehensive set of on-line diagnostics, which are complementary each other and can be used simultaneously, is a very powerful tool for laser plasma interaction studies. In each experiment only various diagnostics would allow unambiguous demonstration of ion acceleration processes in their whole complexity, providing a set of data also for theoretical interpretation. 


\section{Specific features of accelerated ion and electron dynamic}

\subsection{Dips in ion emission spectrum and electron dynamics}

Several physical mechanisms have been considered for the appearance of high-energy electrons, and have been proposed as a way to understand the generation of ions with kinetic energies of several tens of $\mathrm{MeV}$ during the short laser-pulse interaction with dense plasmas, (Malka \& Miquel, 1996, Clark et al., 2000b, Forslund \& Brackbill, 1982, Fews et al., 1994, Beg et al., 1997, Gitomer et al., 1986). A theory has been developed (Wickens et al., 1978, Wickens \&.Allen, 1979) for the free expansion of the laser plasma with hot $\left(T_{h}\right)$ and cold $\left(T_{c}\right)$ electron temperature components, as a way to treat these non-equilibrium effects and to describe the ion energy distribution. It was shown that the energy fraction carried by fast ions depends on the temperature and concentration of the electrons in the plasma. This leads to an ion-emission velocity spectrum whose most notable feature is a pronounced dip

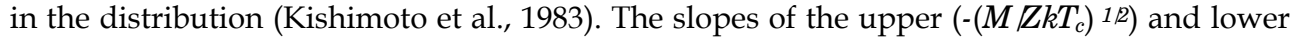
$\left(-\left(M / Z k T_{h}\right)^{1 / 2}\right)$ asymptotes in the ion velocity spectrum makes possible a determination of the effective absolute hot- and cold-electron temperatures (Wickens \&.Allen, 1981). (Here, $M$ is ion mass, $Z$ is charge state, $k T$ is cold- or hot-electron energy).

The dip in the velocity distribution corresponds to an internal electrostatic sheath appearing due to hot- and cold-electron isothermal expansion, where ions are strongly accelerated in a small region. This dip develops in a region of self-similar flow where the ions experience rapid acceleration due to an abrupt increase in the electric field. This increase occurs at the location in the expanding plasma where most of the cold electrons are reflected, corresponding to a step in the ion charge density (Wickens \&.Allen, 1981). The depth of the dip as a function of the peak field is a sensitive function of the hot-to-cold electron temperature ratio $T_{h} / T_{c}$ in the ion spectra, while the position in the spectrum depends on the hot-to-cold electron density ratio $\mathrm{n}_{h} / n_{c}$.

In a very short pulse ( $35 \mathrm{fs}$ ) and high intensity laser plasma several groups of electrons with different temperatures can be generated (Zhidkov et al., 2001), which could then cause multiple dips in the ion energy spectra. No hint of this has been observed so far, and therefore detailed measurements of the ion and electron spectra are required to find correlations between these processes.

Here we report on precise measurements of the spectral density distribution of the ion emission from plasmas created by $35 \mathrm{fs}$ laser pulses at intensities of $(0.8-1.2) \times 10^{19} \mathrm{~W} / \mathrm{cm}^{2}$. A pulse from a multi-TW Ti: Sapphire laser (Kalachnikov et al., 2002) with a maximum energy of $750 \mathrm{~mJ}$, was focused with an $\mathrm{f} / 2.5$ off-axis parabolic mirror onto water droplets of $\sim 20$ $\mu \mathrm{m}$ diameter (Hemberg et al., 2000). Measurements were made of ion spatial and energy distributions, X-ray spectral properties, the electron spectrum emitted, and the correlation of maximum electron energies with the cut-off energies of proton and deuteron spectra.

The ion energies have been measured with a Thomson parabola spectrometer, into which the ions enter through a $200 \mu \mathrm{m}$ aperture. The ions are detected by a $40 \mathrm{~mm}$ MCP coupled to a phosphor screen. The signal from the phosphor screen is imaged with a charge-coupled device (CCD- camera). An ${ }^{241} \mathrm{Am} \alpha$-particle source (energy $5.4 \mathrm{MeV}$ ) with known emittance is used to calibrate the setup (Ter-Avetisyan, et al., 2005). This sensitive, calibrated detection technique allows the measurement of an ion spectrum from a single laser shot in absolute terms.

A typical camera picture taken with a single laser shot, showing ion traces from a heavy water droplet, is depicted in Fig. 2 and the deduced deuteron spectra is shown in the inset. 


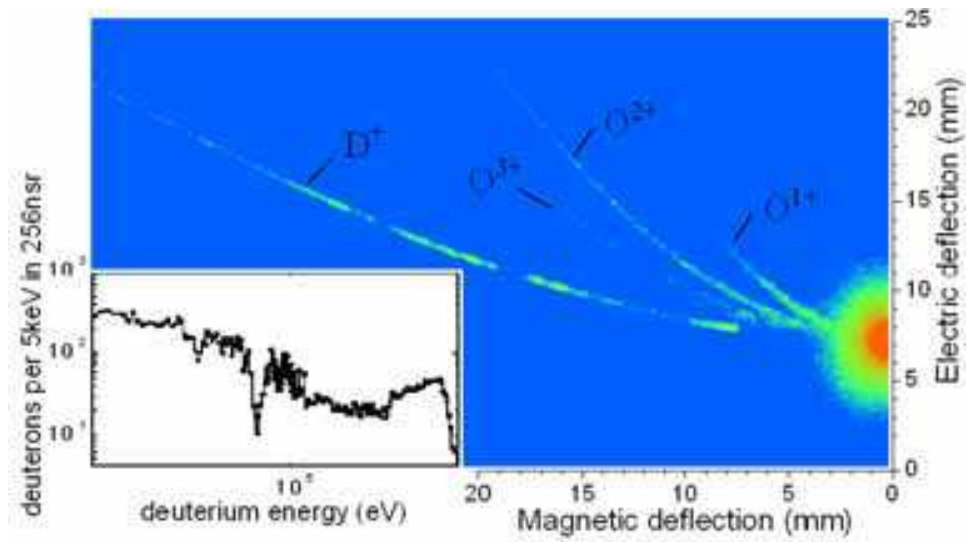

Fig. 2. Color image from the MCP-phosphorous screen of an emitted ion spectra (deuterons and oxygen ions, right blob - "zero" point: radiation impact along spectrometer axis) from a heavy water droplet taken from a single laser shot in backward (1350 to laser propagation) direction with the Thomson spectrometer. In inset the deuteron spectrum is plotted.

The most interesting features in the picture are the clearly visible dips along the deuteron trace. As mentioned before, the same feature has been observed for proton emission from water droplets, and has been observed in backward ( $135^{\circ}$ to the laser axis) as well as in forward (laser propagation direction) emission. The occurrence of the spectral dips was reproducible in the experiment, although the exact position, depth, and fine structure varied from shot to shot due to small variations in the laser parameters and beam alignment in our setup. This is, to our knowledge, the first observation of these dips in ultra-short (sub $50 \mathrm{fs}$ ) high intensity laser-plasma interaction experiments.

The generated hot electron spectrum was measured with a GAFchromic film (HD-810) in a direction transverse to the laser axis using a $0.27 \mathrm{~T}$ magnet spectrometer. The film is sensitive to electrons above $10 \mathrm{keV}$ due to its layer characteristic (Busch, et al., 2003). The measured time-integrated hot electron spectrum (Fig.3a) shows a maximum at an energy of about $0.7 \mathrm{MeV}$ with a tail expanding to 2 or $3 \mathrm{MeV}$.

From the measured electron spectra, one can deduce a hot electron component with a temperature of $(0.63 \pm 0.03) \mathrm{MeV}$. This fits well with the energy the electrons can acquire from the ponderomotive force $F_{p}$ of the laser pulse (Kruer \& Estabrook, 1985): $F_{p}=-d U_{p} / d z, U_{p}(\mathrm{eV})=9.33 \times 10^{-14} I\left(\mathrm{~W} / \mathrm{cm}^{2}\right) \lambda^{2}(\mu \mathrm{m})$, where $U_{p}$ is the ponderomotive energy. This gives a potential energy of $0.6 \mathrm{MeV}$ for our laser intensity, proving a rather efficient laser energy transfer to the electrons. Because up to $20 \%$ of the laser energy can be absorbed in energetic electrons (Wilks et al., 1992), a significant number of electrons with energies of several hundreds of $\mathrm{keV}$ is produced. The electron impact ionization cross section at the energies $400-500 \mathrm{keV}$ is about $10^{-19} \mathrm{~cm}^{-2}$ (http://physics.nist.gov/cgibin/Ionization/table.pl?ionization=H2O), therefore these highly energetic electrons can cross the target without being significantly slowed. A space-charge field accumulated in the droplet captures the hot electrons with energies below about $200 \mathrm{keV}$ (low energy cut off in Fig.3a). The potential due to these electrons and the estimated electron density is sufficient to create electrostatic acceleration fields of the order of $1 \mathrm{MV} / \mu \mathrm{m}$ (Busch, et al., 2003) which, in turn, can accelerate ions to $\mathrm{MeV}$ energies. Electrostatic or magnetic fields around the 
target will influence the directionality of ion emission. However, for our spherical target, we expect a relatively isotropic ion emission distribution, which can be shown by measurements (Busch, et al., 2003, Karsch et al., 2003).

A strong correlation between the maximum ejected electron energies and the deuteron cutoff energies could be directly established (Fig.3b) with the help of a re-designed Thomson spectrometer allowing to measure in one laser shot the emitted ion and electron spectrum in a same direction. For that the second MCP detector was added to that side of the spectrometer where the electrons are deflected. In Fig.3b, a variation in the maximum energies of the ejected electrons by only a factor of 1.2 changes the deuteron cutoff energy by a factor of about 5 . This extreme sensitivity emphasizes the predominant role of the energy transfer to the electrons for the ion acceleration.

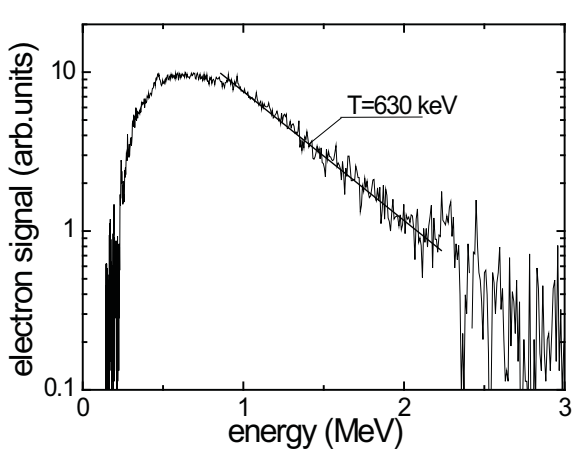

(a)

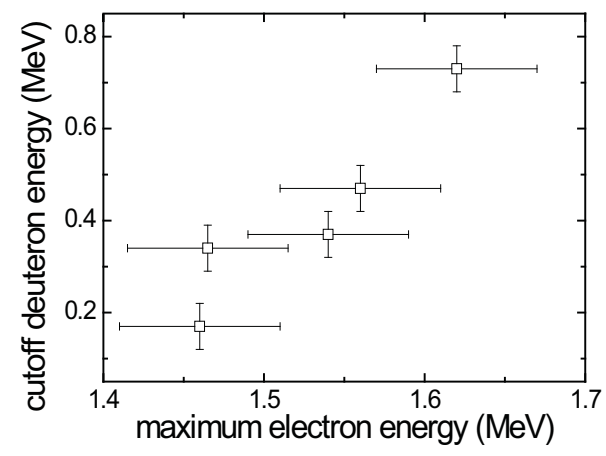

(b)

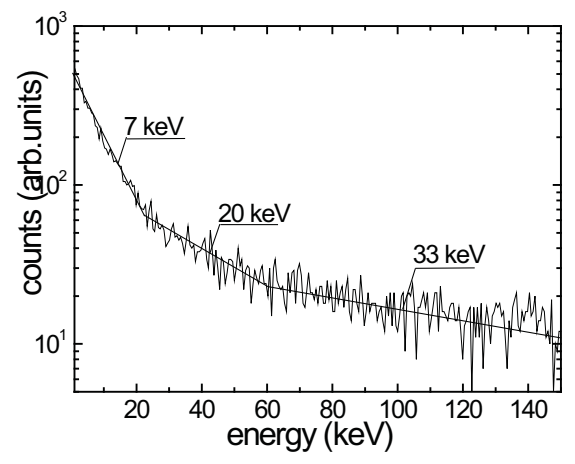

(c)

Fig. 3. Electrons, ions and x-rays emitted of heavy water micro droplet. a) Hot electron energy distribution (superposition of $\sim 50000$ shots). Slope fits to an exponential decay with a temperature $\mathrm{T}=630 \mathrm{keV}$ parameter. $\mathrm{b}$ ) Correlated maximum energies of emitted electrons and deuterons in same laser shot. c) X-ray emission spectra from heavy water droplet.

The space charge field built up by the trapped hot electrons in the target is responsible for ion acceleration properties (Fig.3a), therefore, these electrons have been studied further in details. According to a simple bremsstrahlung model (Griem, 1964), hard X-ray emission 
from the plasma is defined by the electron density distribution inside the target. For highly energetic electrons crossing the droplet, the target is "thin", so their bremsstrahlung is weaker and the spectral intensity is constant (Blochin, 1957).

A calibrated X-ray CCD-camera operating in a single-photon detection mode was used for energy-dispersive X-ray measurement (Ter-Avetisyan, et al., 2003). In the experiments, the CCD-camera was mounted at an angle of $90^{\circ}$ to the laser propagation direction and at a distance of $100 \mathrm{~cm}$ from the plasma source. A $200 \mathrm{~nm} \mathrm{Zr}$ filter and a beam aperture was used to block the optical light and the scattered X-rays. The resolution of our spectral diagnostics is about $0.5 \mathrm{keV}$. A typical X-ray spectrum is shown in Fig.3c. The slope of the distribution shows the existence of multi-temperature components, and can be fitted assuming three different effective temperatures of about $(7 \pm 0.3) \mathrm{keV},(20 \pm 4) \mathrm{keV}$, and $(33 \pm 12) \mathrm{keV}$.

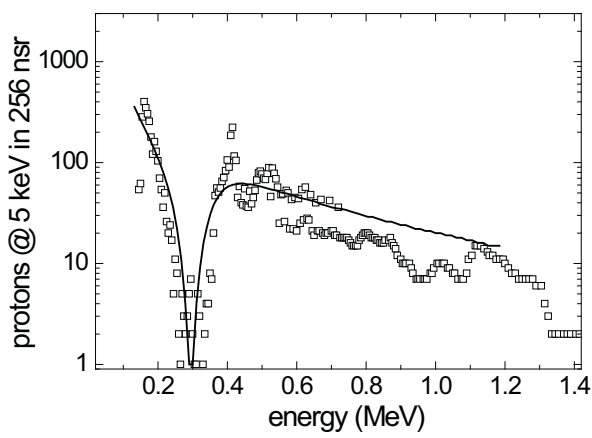

(a)

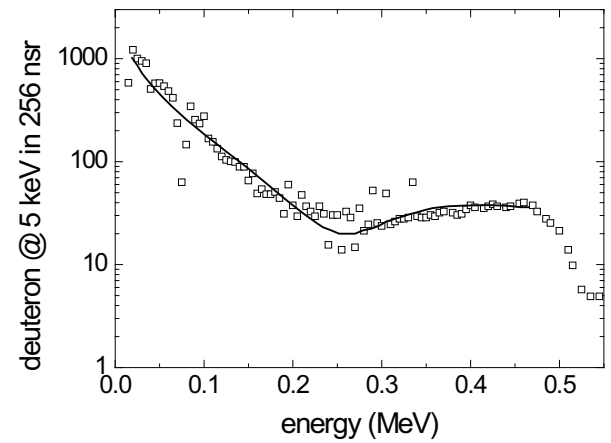

(b)

Fig. 4. Single shot proton a) and deuteron b) spectrum with typical dips along the trace: squares - experiment, line - simulation (parameter cf. text)

Recently, a fluid model based on a single electron temperature approximation was applied successfully for high intensity laser-driven ion acceleration (Mora, 2003). Accurate results could be obtained for the structure of the ion front, the ion energy spectrum, and the cutoff ion energy. In the present letter, on the other hand we explain the dips in the emitted ion spectrum (Fig.2) by relying on the fact that we have an electron spectrum characterized by several electron temperatures. This is the precondition for an application of the fluid model (Wickens et al., 1978, Wickens \&.Allen, 1979, Kishimoto et al., 1983). Figures 4a and 4b compare experimental proton and deuteron energy distributions with calculations based on the theory of Wilkens et al. (1978). A reasonable fit for the depth and position of the dip in the proton spectrum (Fig.4a) is obtained when the hot-to-cold electron temperature ratio $T_{h}$ $T_{c}$ is assumed to be about 9.8 , and the hot-to-cold electron density ratio $n_{\mathrm{h}} / n_{\mathrm{c}}$ is about $1 / 100$. Individual electron temperatures $T_{c}=7.5 \mathrm{keV}$ and $T_{h}=74 \mathrm{keV}$ compare quite well to the range of temperatures derived from the X-ray emission. Here, $T_{h}$ is lower by a factor of about 2, but this can be due to the restricted linearity range $(<50 \mathrm{keV})$ in the X-ray measurement. Also, if one takes into account that bulk ion energy scales with the hot electron temperature as $E_{\text {ion }}=4.5 T_{h}$ (Wickens \&.Allen, 1979), a mean ion energy $E_{\text {ion }}=330$ $\mathrm{keV}$ would be derived, in remarkably good agreement with the ion temperature inferred from the ion slope (Fig.4a). This is somewhat different from the original model where the ion energy is predicted to be similar to the hot cf. cold electron energies. A sharp proton 
cutoff energy occurs at about $1.3 \mathrm{MeV}$. Notice that the analytical velocity distribution derived in (Wilkens et al. 1978) breaks down if the temperature ratio $T_{h} / T_{c}$ exceeds 9.9. In order to overcome this problem, a more complex electron velocity distribution was included in (Kishimoto et al., 1983) [33].

For a heavy water droplet, presented in Fig. $4 \mathrm{~b}$ where the cutoff energy occurs at about 0.55 $\mathrm{MeV}$, the model with a hot-to-cold electron temperature ratio $T_{h} T_{c}=7.7$ and an electron density ratio $\mathrm{n}_{h} / n_{c}=1 / 25$ fits the measurement quite well. The individual temperatures are $T_{c}=11 \mathrm{keV}$ and $T_{h}=85 \mathrm{keV}$. The model shows how small changes in $T_{\mathrm{e}}$ and $n_{\mathrm{e}}$ have large effects on spectral slopes.

From the structure of the emitted ion spectrum such important parameters as hot- and coldelectron temperatures and their density ratio can be determined. Due to the short laser pulse the hot electron population shows a multi-temperature behaviour. This, in turn, can cause multi-dips in the ion spectrum. It is worth noting that the results demonstrated here could open a way to tailor the ion spectra (Nishihara at al., 2001) from short pulse laser-driven plasmas by choosing proper electron distributions appropriate to particular applications.

\subsection{Laser pulse contrast and electron dynamic}

Two different laser energy absorption mechanisms at the front side of a laser-irradiated foil have been found to occur, such that two distinct relativistic electron beams with different properties are produced. One beam arises from the ponderomotively driven electrons propagating in the laser propagation direction, and the other is the result of electrons driven by resonance absorption normal to the target surface. These properties become evident at the rear surface of the target, where they give rise to two spatially separated sources of ions with distinguishable characteristics when ultra-short (40 fs) high-intensity laser pulses irradiate a foil at $45^{\circ}$ incidence. The two, clearly distinguishable branches of electron trajectories have been measured by Čerenkov diagnostics. This correlates with proton emission from two separated sources, which have been resolved with a high-spatialresolution Thomson spectrometer (Schreiber et al., 2006). The crucial parameters of the experiment are the laser pulse intensity and the contrast ratio.

In the experiments, a 40 fs pulse from a multi-TW Ti: Sapphire laser was focused onto a thin $(6 \mu \mathrm{m})$ aluminum foil target at $45^{\circ}$ with an intensity of about $2 \times 10^{19} \mathrm{~W} / \mathrm{cm}^{2}$. The temporal contrast of the laser pulse was characterized by a scanning third-order cross correlator with a dynamic range of $10^{10}$, having a resolution of $150 \mathrm{fs}$ and a scanning range of $\pm 200 \mathrm{ps}$. The pulse shape several ns before the main pulse was controlled by a fast photodiode with temporal resolution of about 300 ps. In typical operating conditions, the amplified spontaneous emission (ASE) pedestal of the laser pulse, several picoseconds before the pulse peak, was at a level of $(0.8-5) \times 10^{-7}$ relative to the peak intensity. This is termed "low" contrast. The ASE pedestal could be reduced by driving the Ti: Sapphire laser amplifiers in specific delayed pump modes. This led to a reduction of pulse energy by up to $550 \mathrm{~mJ}$, but it permitted an improvement of the ASE level down to $(1-3) \times 10^{-8}$, which is referred to as "high" contrast. In both cases, no pre-pulses were observed.

The Čerenkov method, applied as an electron diagnostic, uses the partial conversion of the electron bunch energy into a flux of photons in a medium where the electron velocity is higher than the light velocity (Stein et al., 2004). A $50 \mu \mathrm{m}$ tesa foil (polypropylene with chemical composition $\left(-\mathrm{C}_{3} \mathrm{H}_{6}-\right)_{n}$, index of refraction - 1.49) used as a Čerenkov radiator was attached with very thin Acrylat glue to the target rear, and imaged with an objective $(\mathrm{f}=8$ $\mathrm{cm}$ ) and a magnification of about 15 to a gated charge-coupled device (CCD) camera. It is 
assumed that a $50 \mu \mathrm{m}$ path length in this low $\mathrm{Z}$ substance has a negligible effect on the energy and main direction of the electrons. Propagating through the foil, the electron beam undergoes filamentation (Manclossi et al., 2006), but nonetheless with unaltered beam directions emerging from the target. To block any light from the laser pulse and its harmonics, a $6 \mathrm{~mm}$ Schott BG18 filter and a $3 \mathrm{~mm}$ VG8 filter were inserted into the beam path. These filters yield a spectral window centered at $510 \mathrm{~nm}$ with a half width of $70 \mathrm{~nm}$. A weak and uniformly distributed background signal from transition radiation is omitted as a result. For the medium and the geometry used, the detected Čerenkov light is associated with electrons in the $180-240 \mathrm{keV}$ energy interval.

Measurements of the ion energies were carried out with a Thomson spectrometer (TerAvetisyan et al., 2005). In order to achieve high spatial resolution, we set up a Thomson spectrometer in a 1:12 imaging mode (Schreiber et al., 2006), providing spatial resolution of about $20 \mu \mathrm{m}$. This estimation takes into account the laminarity of the proton beam, and is measured from the spreading of a spectral trace which is a consequence of the divergence of the proton beamlet intercepted by a screen with a $30 \mu \mathrm{m}$ pinhole.

The Čerenkov medium behind the target made it impossible to use both diagnostics simultaneously for the single laser shot. Therefore, correlations between the data sets are statistically relevant. Due to a $10 \mathrm{~Hz}$ repetition rate of the Ti:Sapphire laser, and using online detection systems for both diagnostics, the statistical uncertainties could be minimized.

Figure 5 shows spatially resolved CCD pictures of Čerenkov radiation behind the $6 \mu \mathrm{m} \mathrm{Al}$ target at $45^{\circ}$ laser incidence $(\mathrm{a}-\mathrm{c})$, and it shows the evolution of the Čerenkov signal as a function of target thickness (d). The arrows inserted in the pictures show the target irradiation and Čerenkov emission geometry. In the pictures, one of the two components of the hot electron current produced is identified as a consequence of hot electrons driven along the target normal due to resonance absorption, and the other component along the laser propagation axis is due to the ponderomotive force. Whereas in Fig.5a both electron components make a comparable contribution to the resulting Čerenkov emission, resonantly and ponderomotively driven electrons are dominant in Figs. $5 b$ and $5 c$, respectively. The appearances of the emission patterns are statistical, and correlated with the statistics of the laser pulse contrast shot-to-shot fluctuation data.

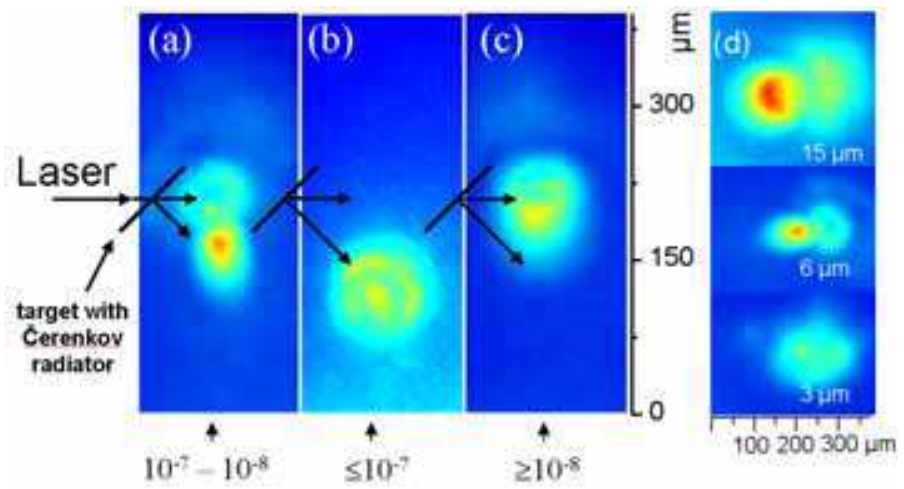

Fig. 5. CCD pictures of Čerenkov radiation (arrows show emission direction) at 45o laser incidence resulting from electrons propagating: (a) in the laser direction and normal to the target; or (b) perpendicular to the laser direction only; and (c) in the laser direction only. (d) Čerenkov light distributions as a function of Al-target thickness. 
The evolution of the Čerenkov signal as a result of target thickness is shown in Fig.5d. With a $3 \mu \mathrm{m}$ target the two components are merged together, while at $6 \mu \mathrm{m}$ they are clearly separated and at the target thickness is $15 \mu \mathrm{m}$, further broadening of the electron beams propagating inside the target occurs, and blurs the Čerenkov emission. In the experiments reported in Ref. (Stein et al., 2004), these two groups of separately moving hot electrons at the target rear side also were observed. Here we investigate the dynamics of the hot electron populations, and the consequences for the ion acceleration processes at "low" and "high" laser pulse contrasts.

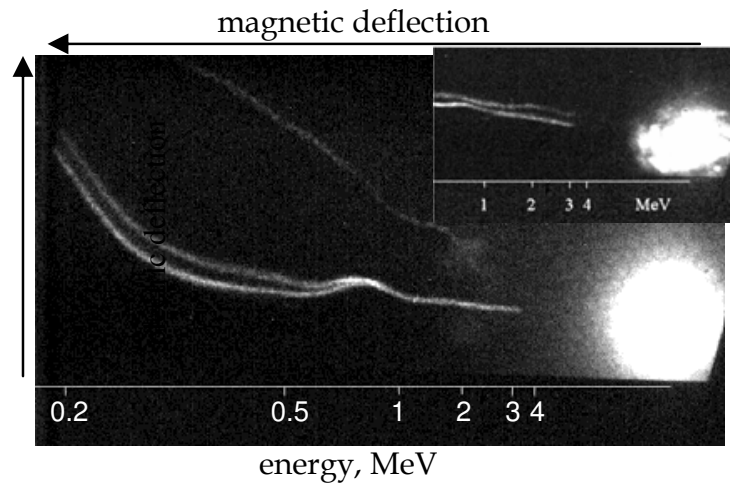

Fig. 6. A CCD picture of a proton spectrum at $45^{\circ}$ laser incidence on a $6 \mu \mathrm{m} \mathrm{Al}$ target, measured in the direction of the target normal. The inset shows proton spectra from a $3 \mu \mathrm{m}$ target thickness at similar irradiation conditions.

The resonantly and ponderomotively driven electron populations at the target rear side create two spatially separated electrostatic fields, from where the ions/protons can be accelerated. Therefore, one can expect ion emission from two spatially separated sources if the laser pulse contrast is between $10^{-8}-10^{-7}$, where both laser energy absorption mechanisms are equivalent. Indeed, the CCD picture of the proton spectrum in Fig.6, measured in the direction of the target normal, shows two parallel proton parabolas, thus confirming the existence of the two sources of proton emission. The lower trace results from the source created by electrons from resonance absorption, and the upper one from the source created by ponderomotively driven electrons. The appearance of the second source (or second proton trace) is in good agreement with the detection of two electron populations by Čerenkov diagnostics (Fig.5a). This feature is never observed at normal laser incidence on the target. Figure 6 also shows that the parabolas are not perfect. This coincides with the previous findings (Schreiber et al., 2006) that the source position could be variable. Note that the two parallel traces in Fig.6 change in a similar manner, while the proton spectrum of a $3 \mu \mathrm{m}$ thick target (inset of Fig.6) shows an independent character, which indicates a complex source dynamics.

In order to see the differences in the electron acceleration processes and the implications for the following ion acceleration, we performed two-dimensional (2D) particle-in-cell (PIC) simulations of laser irradiation onto targets with different scales of pre-plasmas. The targets are $2 \mu \mathrm{m}$ thick and have densities of $40 \mathrm{n}_{\mathrm{c}}$. The pre-plasma is assumed to have an exponentially decreasing profile with two scale lengths. For the overdense region where the density is higher than $4 \mathrm{n}_{\mathrm{c}}$, the scale length is $0.1 \mu \mathrm{m}$. For the lower density region resulting from an expanding 
corona plasma, the scale lengths are $0.3 \mu \mathrm{m}$ for the small pre-plasma case and $3.5 \mu \mathrm{m}$ for the large pre-plasma case. The initial electron temperature is $1 \mathrm{keV}$, and ions are kept immobile. The targets are irradiated by p-polarized laser pulses from the left boundary with wavelengths of $1 \mu \mathrm{m}$. The laser pulses ramp up in five laser cycles, and sustain a peak intensity of $2 \times 10^{19}$ $\mathrm{W} / \mathrm{cm}^{2}$ that is uniform in the $\mathrm{y}$-direction. The pulse duration is $40 \mathrm{fs}$. The boundary conditions are absorbing in the $\mathrm{x}$-direction, and periodic in the $\mathrm{y}$-direction.

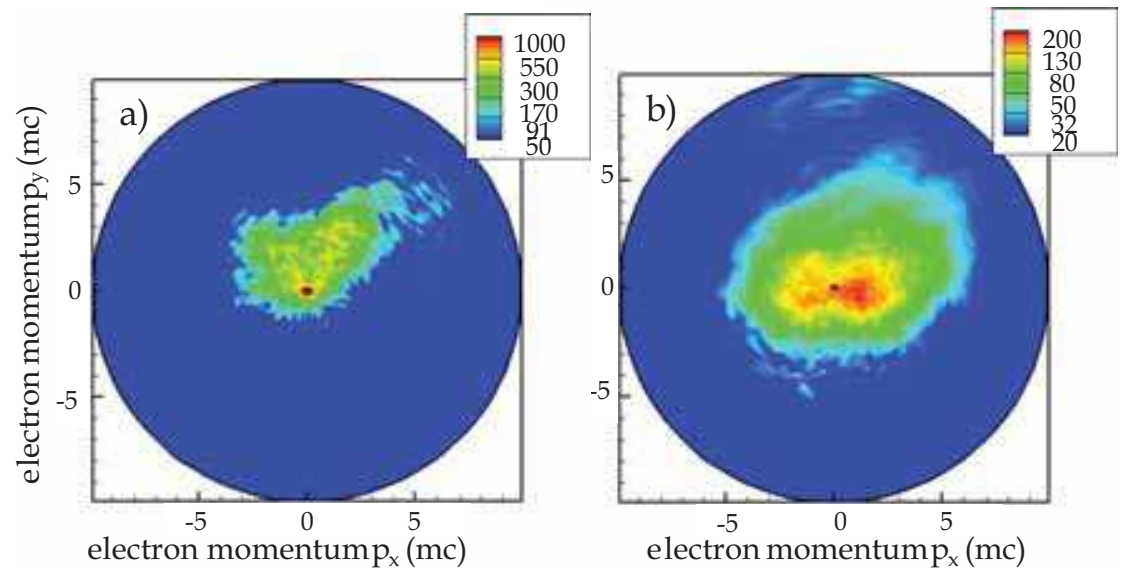

Fig. 7. Electron momentum distribution observed in PIC simulation at the target rear surface for a) "small" $\mathrm{L}=0.3 \mu \mathrm{m}$ scale length pre-plasma case, and for $\mathrm{b}) \mathrm{L}=3.5 \mu \mathrm{m}$ "large" scale length pre-plasma case. Laser pulses irradiate the target from the left boundary.

The differences resulting from the dominant acceleration processes are seen from the angular distribution of accelerated electrons. In Fig.7, the angular electron momentum distributions observed in the PIC simulation at the target rear surface are shown for the a) "small" $\mathrm{L}=0.3 \mu \mathrm{m}$ scale-length pre-plasma case, and for the b) $\mathrm{L}=3.5 \mu \mathrm{m}$ "large" scale-length pre-plasma case. In the small pre-plasma case, ponderomotive acceleration and vacuum heating play dominant roles in energy absorption by electrons. Due to vacuum heating, laser energy is absorbed by low energy bulk electrons that are mainly accelerated in the target-normal direction. High energy electrons generated by ponderomotive acceleration propagate in the laser irradiation direction, and are reflected back toward the target at the specular angle, as can be seen clearly in the figure. For the large pre-plasma case, high energy electrons are mainly accelerated by resonance absorption in the target normal direction and are reflected back also in the normal direction.

These high energy electrons induce a strong magnetic field around the critical surface. In the small pre-plasma case, a magnetic field with uniform intensity is induced along the critical surface. This surface magnetic field prevents low energy electrons from propagating into the target, and makes them flow along the surface (Nakamura et al., 2004). In the large preplasma case, a magnetic field is formed via Weibel instability (Weibel, 1959), which is in the target-normal direction. The surface field tends to make electrons flow along the surface, which leads to a uniform structure of the interaction surface. The target-normal magnetic field acts to collimate electron jets and return currents that are periodic along the surface. As a result, the critical density surface is rather smooth in the small pre-plasma case and modulated in the large pre-plasma case. 


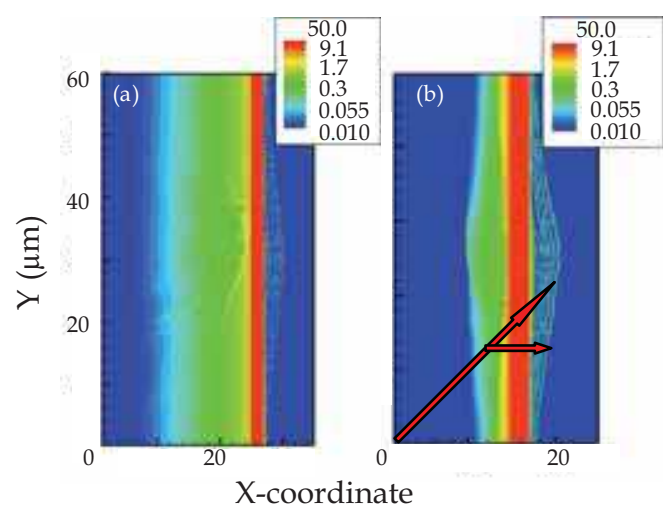

Fig. 8. Ion density distribution for a) large $(\mathrm{L}=3.5 \mu \mathrm{m})$ and $\mathrm{b})$ small $(\mathrm{L}=0.3 \mu \mathrm{m})$ pre-plasma cases. Laser irradiation is from the left boundary, where the spot size is $15 \mu \mathrm{m}$, and the spot centre corresponds to $y=8.5 \mu \mathrm{m}$ at the left boundary. Two arrows in $\mathrm{b}$ ) indicate the trace of the laser spot centre and the target-normal projection of the spot centre.

These different electron acceleration processes lead to differences in the lateral distributions of the rear side sheath field and proton acceleration as follows. The target size is $2 \mu \mathrm{m}$ in thickness and $50 \mu \mathrm{m}$ in width. The targets consist of $50 \%$ protons and $50 \% \mathrm{C}^{4+}$. Laser conditions are the same as above except that the laser pulses have a spot size of $15 \mu \mathrm{m}$ with a super-Gaussian distribution. The boundary conditions are absorbing in the $x-$ and $y-$ directions. Ion density profiles at $150 \mathrm{fs}$ after the pulse has ended are shown in Fig.8.

For the large pre-plasma case, the ion distribution is symmetric with respect to the laser axis passing through the laser focal point at the critical surface. Since a large part of the hot electrons propagate in the target-normal direction, the sheath field structure is similar to the normal incidence case. For the small pre-plasma case, however, the proton distribution is non-uniform and asymmetric. As is seen in previous simulations, very energetic electrons are accelerated in the laser propagation direction, and lower energy electrons are generated in the target-normal direction via vacuum heating. These two components result in two acceleration fronts of protons, which are clearly seen in Fig. $8 \mathrm{~b}$ and detected in the experiment. The distance between the two acceleration points depends on target thickness and size of pre-plasma. In PIC simulation, the target is thinner and the density is lower than in the experiments. Thus the separation distance is considered to be larger than is visible in PIC simulation. Also, in Fig.8 we show the angular distributions for pre-plasma scales of 0.3 and $3.5 \mu \mathrm{m}$ in order to see clear differences, but similar results are seen at much smaller differences also. The important thing is that the dominant acceleration process changes when the scale length becomes smaller than the wavelength.

A PIC simulation of the experimental parameters shows that the dominant electron acceleration process depends on the profile of the pre-plasma. For plasmas where the scale length of the pre-plasma is larger than the laser wavelength, a plasma wave is resonantly excited at the critical density surface, which results in the generation of fast electrons propagating in the target-normal direction (Estabrook \& Kruer, 1978). If the pre-plasma scale length is smaller than the laser wavelength, a well-collimated electron beam is accelerated by ponderomotive forces in the propagation direction of the laser (Wilks et al., 1992, Kruer \& Estabrook, 1985). Correspondingly, protons are accelerated in a target-normal 
direction from two spatially separated sources. The laser intensity and the contrast determine the laser absorption process and thus they control the entire acceleration scenario. PIC simulations confirm these findings.

\subsection{Energy resolved spatial characteristics of laser accelerated protons}

As we have seen, interactions of relativistic-intensity laser pulses with targets with and without pre-plasmas are significantly different from the perspective of the dominant electron acceleration processes and the energy coupling to plasmas.

To clarify the proton acceleration process that is uniquely observed for small pre-pulses, oblique irradiation, and in thin targets we incorporate particle diagnostics which provide a microscopic measurement of the proton acceleration with PIC simulations. We found that the laser-accelerated proton source drifts on the rear surface of the target in the direction of projection of the laser wave vector onto the surface. The drift of the proton source is explained by the lateral movement of the sheath field. This moving proton source leads to the observation of a limited range of proton energies at any given point along the target surface.

The proton source was investigated with an experimental setup and geometry similar to those in section 1.2. The ion emission spectrum was measured with an absolutely calibrated Thomson parabola spectrometer (Ter-Avetisyan et al., 2005) set up in magnifying imaging mode (Schreiber et al., 2006). The spectrum is imaged through a $30 \mu \mathrm{m}$ pinhole, located at a distance of $5 \mathrm{~cm}$ from the source, on a detector screen $75 \mathrm{~cm}$ behind the pinhole, at $45^{\circ}$ laser incidence on a $13 \mu \mathrm{m}$ aluminum target; see Fig.9.
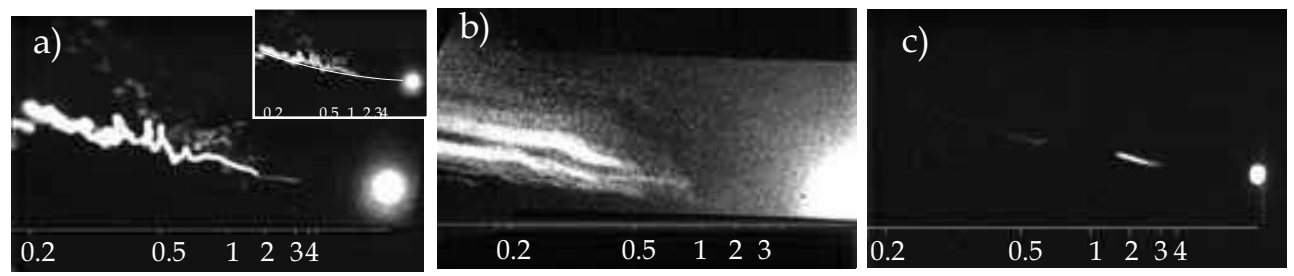

d)

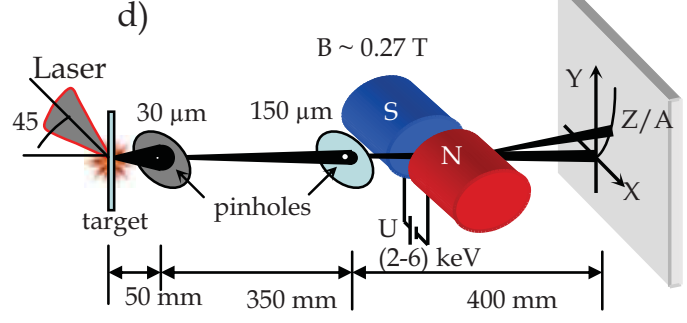

Fig. 9. a), b), c) CCD picture of the proton spectrum at $45^{0}$ laser incidence on a target measured in the direction of target normal with a magnified imaging ratio of 1:15. The ideal parabola trace is the solid line in the figure a) inset. b) Proton spectrum from a Mylar target. c) Proton spectrum from $\mathrm{Al}$ target when proton beam passes through two pinholes. $\mathrm{d}$ ) Schematic figure of the geometry of two pinhole measurement. 
The striking features of this figure are that the whole low-energy part of the spectrum is shifted from the ideal parabolic trace calculated according to our geometry, whereas the high-energy part obeys the calculated parabolic trace (solid line in Fig.9a inset) assuming that the ions are emitted from a fixed ion source, and finally that the trace of the low-energy part shows strong oscillations.

The imaging factors of the setup include the geometry and the emitted proton beam characteristics, such as partial beam divergence. In Fig.9a it is found that the trace spread decreases with increasing proton energy. The trace spread and the partial beam divergence calculated for the present geometry (distance from the source to the detector) decrease from 0.13 to $0.11 \mathrm{mrad}$ when the proton energy increases from 0.2 to $3 \mathrm{MeV}$. Therefore, in the present geometry, the image covers only the diameter of an $18 \mu \mathrm{m}$ area on the target with a magnification factor of about 15. The observed phenomena are attributed to the continuous movement of the proton emission source up to about $20 \mu \mathrm{m}$. When electron beam filamentation occurs during electron propagation inside the target, as it is the case for insulators (Manclossi et al., 2006, Fuchs et al., 2003), several proton emission points and, correspondingly, multiple parabolic traces would be measured. The proton spectra from 13 and $20 \mu \mathrm{m}$ Mylar targets taken at a relatively low laser pulse contrast $\left(\sim 10^{-7}\right)$ indicate many separate proton emission points on the target. In Fig.9b is given, as an example, a proton spectrum from a $20 \mu \mathrm{m}$ Mylar target. Here the energy scale is calculated assuming that the source point is in the middle of the right blob in Fig.9b.

The continuous proton spectrum in Fig.9a suggests that one proton source point is continuously moving on the surface. In order to give additional proof of this assumption, a second pinhole with a diameter of $150 \mu \mathrm{m}$ was installed at $35 \mathrm{~cm}$ behind the first pinhole (see Fig.9d), in order to detect only the protons emitted from an area of $20 \mu \mathrm{m}$ diameter with straight trajectories along this axis. Particles that are emitted from the other parts of the source and even have passed the first pinhole will be blocked by the second one. A continuous decrease of the size of the second pinhole led to truncation of the spectrum and finally only the high-energy part of the spectrum remains, which lies on the expected ideal parabolic trace as shown in Fig.9c, indicating that they are accelerated from the indicated point source. Here, another interesting point is that the source position from which the high energy protons are emitted does not emit the low-energy ones in the same direction. It has to be mentioned that these phenomena are observed only at $45^{\circ}$ laser irradiation case.

2D PIC simulations were carried out to see the temporal evolution of the sheath field on the rear surface. The simulation conditions are as follows. The targets are $13 \mu \mathrm{m}$ in thickness and $70 \mu \mathrm{m}$ in width with density of $40 \mathrm{n}_{\mathrm{c}}$, where $\mathrm{n}_{\mathrm{c}}$ denotes the critical density for the laser wavelength of $0.8 \mu \mathrm{m}$, and consist of electrons and $\mathrm{Al}^{3+}$ ions. The proton source is a contaminant layer, which is modelled as a buried proton layer at the rear surface with thickness of $0.1 \mu \mathrm{m}$ and density of $1 \mathrm{n}_{\mathrm{c}}$, i.e., the $0.1 \mu \mathrm{m}$ layer from the rear surface is composed of $39 \mathrm{n}_{\mathrm{c}}$ of $\mathrm{Al}^{3+}$ and $1 \mathrm{n}_{\mathrm{c}}$ of protons, with $40 \mathrm{n}_{\mathrm{c}}$ electrons. The electrons initial temperature is set at $1 \mathrm{keV}$, and the ions are initially cold. The system size is $50 \mu \mathrm{m}$ in the $\mathrm{x}$ direction and $70 \mu \mathrm{m}$ in the y direction and the cell number is $2245 \times 3369.12$ particles are used in one mesh for each species and the total number of particles is about $8 \times 10^{7}$. The density profile of the pre-plasma is assumed to be exponential with two scale lengths. For the overdense region where the density is higher than $3 n_{c}$ the scale length is $0.1 \mu \mathrm{m}$. For the lower-density region, lower than $3 \mathrm{n}_{\mathrm{c}}$, the scale length is 0.5 or $3.5 \mu \mathrm{m}$. A p-polarized laser pulse whose spot diameter is $10 \mu \mathrm{m}$ irradiates the target from the left boundary with the 
incident angle of $45^{\circ}$. The laser pulse ramps up in five laser cycles and sustains its peak intensity of $2 \times 10^{19} \mathrm{~W} / \mathrm{cm}^{2}$ for a duration of $40 \mathrm{fs}$. The laser axis irradiates the target surface at $\mathrm{y} \cong 17 \mu \mathrm{m}$. On the boundaries, particles are reflected back to the system by reducing their energy to the thermal one, and the electromagnetic fields are damped at the boundaries.
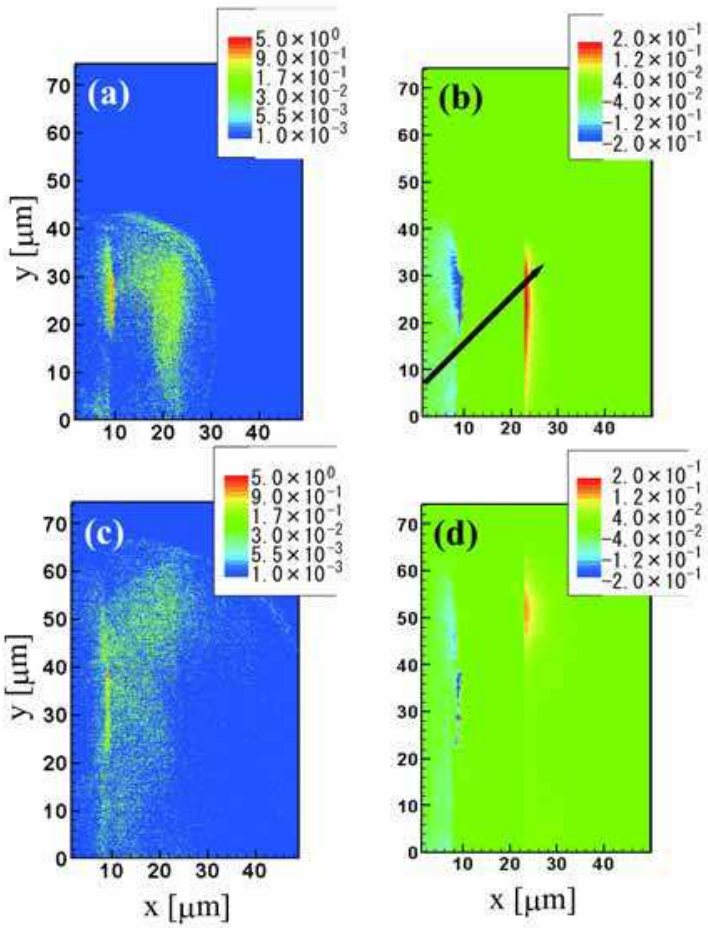

Fig. 10. Temporal evolution of the electron energy density and the longitudinal electric field. The electron energy density is normalized by $\mathrm{n}_{\mathrm{c}} \mathrm{m}_{\mathrm{c}}{ }^{2}$, and observed at a) $140 \mathrm{fs}$, and c) $220 \mathrm{fs}$. The electric field is normalized by laser electric field, and observed at b) $140 \mathrm{fs}$, and d) $220 \mathrm{fs}$. The target width is $13 \mu \mathrm{m}$ and is located at $10 \mu \mathrm{m}<x<23 \mu \mathrm{m}$. The black line in b) indicates the laser axis.

The temporal evolution of the longitudinal electric field $E_{x}$ and electron energy density distribution for the small pre-plasma case $(0.5 \mu \mathrm{m}$ length scale in the lower-density region) are shown in Fig.10. The region where electron energy density is high moves upward; this direction of movement is explained as follows. The high-energy electrons are accelerated in the laser propagation direction by ponderomotive acceleration for the small-pre-plasma case. When they exit from the rear surface, very energetic electrons escape from the target, but a large number of the electrons are dragged back and reinjected into the target toward the front side. As a result, electrons move upward, being trapped along the target and therefore the sheath field also moves in the same direction, as is seen in Fig.10, which results in the movement of the proton acceleration point.

There are two components of the sheath fields showing different time evolutions. The sheath field induced by electrons accelerated in the target-normal direction by vacuum 
heating is symmetric around the target-normal position and expands radially, quickly reducing its magnitude. Another component is generated by electrons accelerated by ponderomotive acceleration, with greater magnitude than the previous one, since the ponderomotively accelerated electrons have much higher energy than target-normal electrons as is shown in Fig.11 and also in Ref. (Sheng et al., 2000).

At the early time of irradiation, a rather symmetrically expanded sheath field is induced. Shortly thereafter, a stronger sheath field localized at the ponderomotive position $(\mathrm{y} \sim 29$ $\mu \mathrm{m})$ is induced, while the symmetric field is weakened by diffusion (Fig.10d). The sheath field is strongest at the ponderomotive position and has a size comparable to the laser spot size.

The temporal evolutions of the maximum of the sheath field intensities at different positions are compared in Fig.12a, where the ponderomotive position (y 29 $\mu \mathrm{m})$, and 11 and $22 \mu \mathrm{m}$ upward to the position (i.e., $y=41$ and $52 \mu \mathrm{m}$ ) are chosen. As is seen from the figure, the intensity of the sheath field decreases as it propagates upward with velocity roughly close to the speed of light. The energy spectra of accelerated protons observed at those positions with $2 \mu \mathrm{m}$ width are shown in Fig.12b. In the figure, the time delay of the proton acceleration due to the sheath movement is taken into account. It is seen that protons with the highest energies $(2.3 \leq \mathrm{E} \leq 3.0 \mathrm{MeV})$ are accelerated at the ponderomotive position by the strong sheath field, and protons accelerated in the upper position by the weakened field have lower energies.

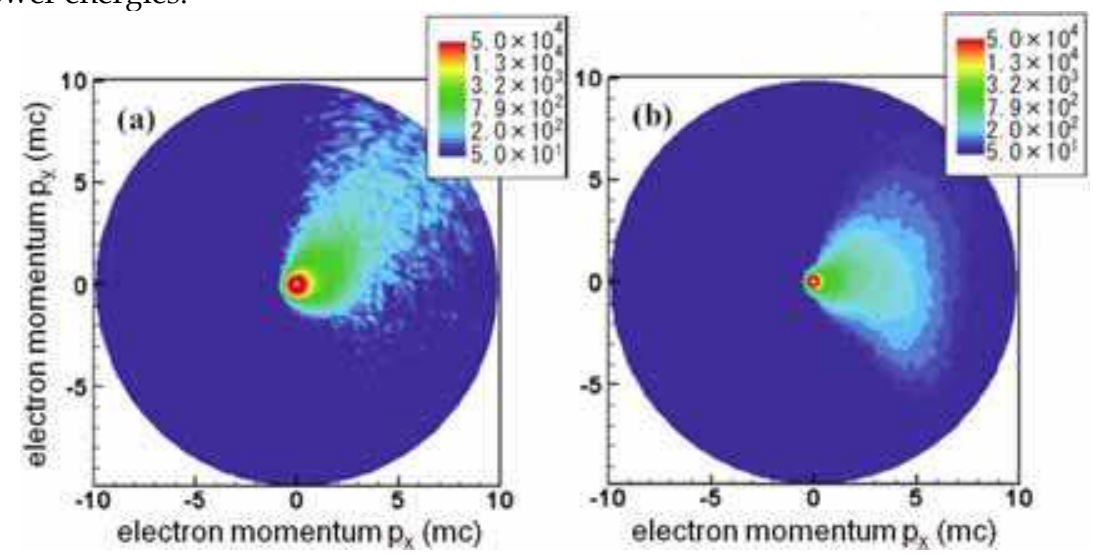

Fig. 11. Angular distribution of laser-accelerated electrons for scale length of preplasma a) 0.5 and b) $3.5 \mu \mathrm{m}$.

Since the lateral movement of the region of high electron energy density is due to the lateral movement of ponderomotively accelerated electrons, the scale length of the pre-plasma is crucial for the drift since the dominant acceleration process depends on the scale length. The angular distributions of electron momentum inside the targets are compared for two different scale lengths, i.e., 0.5 and $3.5 \mu \mathrm{m}$, shown in Figs.11a and 11b. In the small-preplasma case, the high-energy electrons are well collimated and accelerated along the laser propagation direction by the ponderomotive acceleration. On the other hand, in the largepre-plasma case, the electrons are dominantly accelerated toward the target-normal direction by the resonance absorption. In this case, the electrons propagating away from the rear surface return to the target in every direction like a fountain, which results in the 
symmetric expansion of the sheath field. As a result, in the large pre-plasma case, directional lateral drift of the sheath field and proton source movement are not observed.

The target thickness is also an important parameter. The lateral movement of the sheath field becomes less effective as the target thickness increases, since the electron energy density at the target surface decreases due to the expansion of the high-energy electrons into the target. Therefore, the target thickness is chosen to be comparable to or less than the laser pulse length for effective lateral drift of the sheath field. Also, the laser intensity is required to be relativistic so that the ponderomotive acceleration becomes dominant, generating a directional electron beam toward the laser propagation direction. A number of experiments on laser-proton acceleration have been carried out with similar parameters in terms of the laser intensity, irradiation angle, pulse duration, and target material and thickness (Hegelich et al., 2002, Fuchs et al., 2006, Cowan et al., 2004). But lateral drift of the sheath field has not been observed. The critical difference is the level of ASE. In the above cited experiments, the ASE level is mentioned as $\sim 4 \times 10^{12} \mathrm{~W} / \mathrm{cm}^{2}$, and in our experiments the ASE is reduced to $\sim 1 \times 10^{11} \mathrm{~W} / \mathrm{cm}^{2}$ by use of a specifically delayed pump mode with a comparable duration.
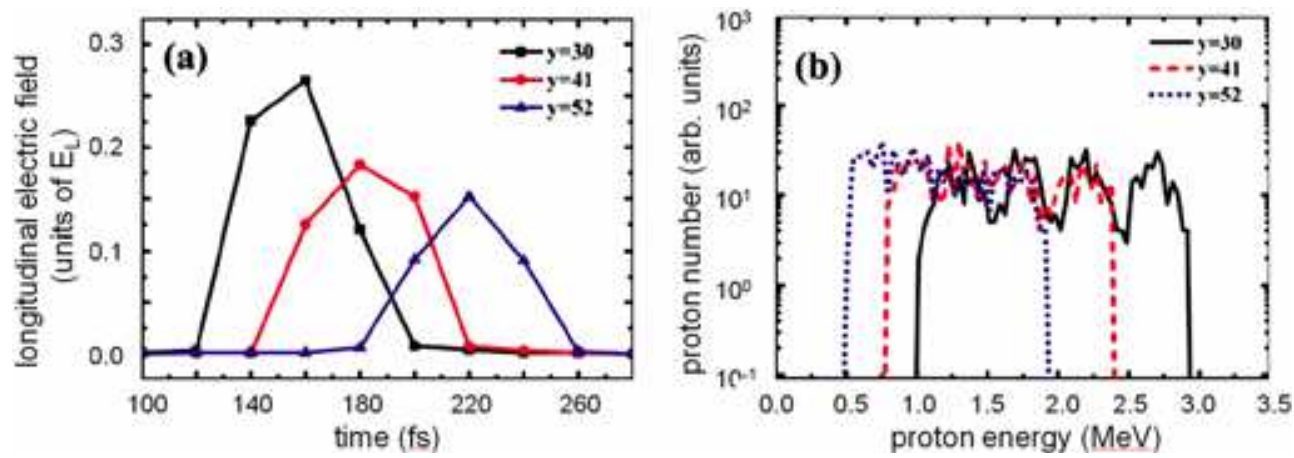

Fig. 12. a) Temporal evolutions of maxima of sheath field intensity at different positions $y=30,41$, and $52 \mu \mathrm{m}$. b) Proton energy spectra observed at three positions chosen the same as in a). The observation timings are delayed for the upper position such that $t=400,440$, and $480 \mathrm{fs}$ at $\mathrm{y}=30,41$, and $52 \mu \mathrm{m}$, respectively, since there is a time delay before the sheath field reaches and starts to accelerate protons.

Thus the amount of energy of the ASE is lowered by roughly $1 / 40$ in our experiments, which leads to the estimated scale length being lowered by $1 / 6$. In the numerical simulations by Fuchs et al., (2006), which show good agreement with the experimental results, the scale length is set at $3 \mu \mathrm{m}$, which is consistent with the scale length of $0.5 \mu \mathrm{m}$ in our experimental conditions. Finally, we want to mention the oscillation in the parabolic trace for the low-energy part. This might be attributable to the complex electron transport coupled to self-induced electric and magnetic fields on the rear surface. In the above simulations, a magnetic field of $\sim 30 \mathrm{MG}$ and electric field of $\sim 1 \mathrm{TV} / \mathrm{m}$ are observed, induced by the lateral transport of high-energy electrons with an estimated current of roughly tens of kiloamperes. This surface current would be disturbed by a Weibel-like instability (Weibel, 1959), whose wave vector lies dominantly in the $\mathrm{z}$ direction, which is not taken into account in a two-dimensional (2D) simulation. Its understanding and consideration of 3D effects are left for future work. 
In conclusion, we have shown a scenario of proton acceleration by a fast-moving rear surface sheath field. This is observed as a directional shift of a single parabolic line, indicating that the proton emission point is drifting along the surface, which is proved by a two-pinhole measurement. This drift is confirmed by 2D PIC simulations which clarify the mechanism and conditions for the drift. The drift is only observed when intense laser pulses obliquely irradiate targets with small pre-plasmas. In this case, ponderomotively accelerated electrons propagate along the target and induce a commoving sheath field. This drift of the acceleration position leads to a selection of the protons by their energies as a function of the observation point, which might shed light on the control of laser accelerated proton energy.

\section{Tomography of an ultrafast laser driven proton source}

\subsection{Correlation of spectral, spatial and angular characteristics of proton source}

Even if the measurements discussed in previous sections show a clear feature of the source, however, those are measurements of the ion emission from a very small area as compared to the whole source extension, which is about a few hundred $\mu \mathrm{m}$. The complete scenario of the ion acceleration phenomena in its complexity could be given only if the source characteristics as a whole or at least a more extended part of it are investigated. Such understanding is especially important for modelling and ion beam tracing.

In order to image a broader area of the source the principle of tomography was employed. Tomography is an imaging method. The object is imaged section by section (or sectioning). In this work we apply sectional imaging of the proton source by using an array of pinholes in combination with a Thomson spectrometer (Ter-Avetisyan et al., 2009a). A technique which provides sectional images of an object is usually referred to as a tomography, and, in principle, the superposition of tomographic cross sections coupled to a reconstruction algorithm allows the visualization of the internal structure of an object.

Because the laser accelerated proton beam is laminar (Borghesi, et al., 2004) and irregularities at small scale do not disrupt the general beam characteristic it is sufficient to substitute the entrance pinhole of the spectrometer with a multi-pinhole array. It is installed perpendicular to the dispersion direction: parallel to magnetic and electric fields. This way the proton beam is divided into small beam-lets and the obtained spectral images combine the simultaneous record of many independent spectrometers which have the same dispersion constant (Fig.13). Because the source has a finite size and the emission is laminar the spectrometers record the ion spectra corresponding to different emission zones of the source according to their emission angle. This detection principle allows the reconstruction of the source, which we call here proton source tomography. It gives an energy resolved topographic picture of the source.

In the experiment $40 \mathrm{fs}$ pulses from a multi $10 \mathrm{TW}$ Ti: Sapphire laser system were focused down to a $10 \mu \mathrm{m}$ perfectly round spot onto a $5 \mu \mathrm{m}$ Ti target. The maximum laser intensity was about $2 \times 10^{19} \mathrm{~W} / \mathrm{cm}^{2}$. The ASE pedestal of the laser pulse, several picoseconds before the pulse peak, was at a level of $10^{-8}$ relative to the peak intensity.

The proton emission spectrum was measured with a Thomson parabola spectrometer calibrated in absolute terms (see more details in Ter-Avetisyan et al., 2005). The applied magnification (Schreiber et al., 2006), provides additionally high spatial resolution. The spectra were obtained from 21 beam-lets produced with a row of 21 pinholes having $30 \mu \mathrm{m}$ in diameter each and a period of $\mathrm{d}=530 \mu \mathrm{m}$ (Fig.13).

This pinhole mask is positioned parallel to target surface at a distance of a $=85 \mathrm{~mm}$, parallel to the magnetic and electric fields of the Thomson spectrometer, and lie in the plane of laser 


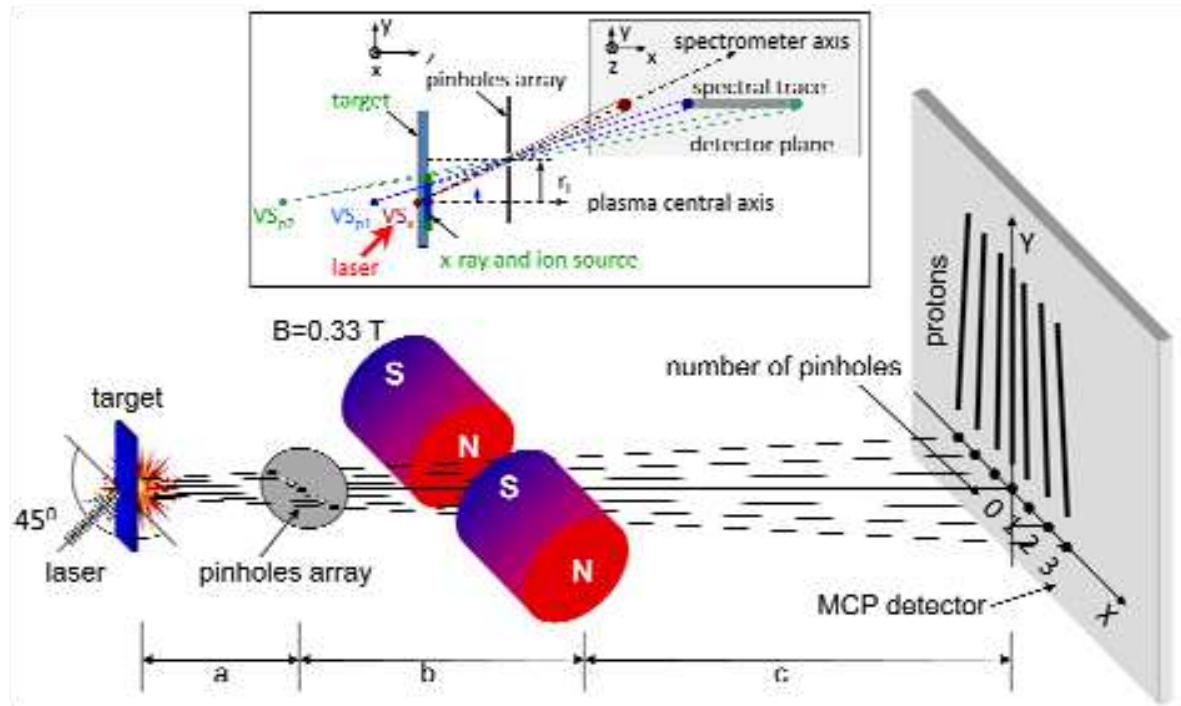

Fig. 13. Experimental set up. Figure inset shows imaging geometry: ions propagate along $\mathrm{Z}$-axis, energy dispersion is along $\mathrm{Y}$-axis, and analyzed emission coordinate is along $\mathrm{X}$-axis. $\mathrm{XY}$ detector plane is flipped in $\mathrm{YZ}$ cartoon plane. Shift of pinholes $\left(\mathrm{r}_{\mathrm{i}}\right)$ and all distances are known from the geometry (cf. text). $\mathrm{VS}_{\mathrm{p} 1}, \mathrm{VS}_{\mathrm{p} 2}$, and $\mathrm{VS}_{\mathrm{x}}$, are the virtual source points for protons with different energies $\left(\mathrm{E}_{\mathrm{p} 1}>\mathrm{E}_{\mathrm{p} 2}\right)$ and $\mathrm{x}$-rays, respectively.

beam polarization (p-polarized). A magnetic field of about $0.33 \mathrm{~T}$ between $4 \mathrm{~cm}$ separated poles was applied in the spectrometer. The spectrum was imaged on the MCP detector coupled to the phosphor screen located at a distance of $b=120 \mathrm{~mm}, \mathrm{c}=275 \mathrm{~mm}$ from the pinholes (Fig.14a). All precautions mentioned in (Ter-Avetisyan et al., 2004b) have been taken for the measurements. Additionally, a careful scan of the magnetic field distribution in all three dimensions between the magnetic poles and outside was carried out. The derived instrument function was conformed with an additional test experiment to exclude any artifact on the spectral traces caused by field inhomogeneities.

At this geometry $\alpha=2.3^{\circ}$ is the largest possible angle of protons entering the spectrometer. The restriction comes mainly due to the finite size of MCP detector. The magnetic field disperses the protons acting on their $v_{z}$ velocity component perpendicular to magnetic field which is $v_{z}=\vec{v} \times \cos \alpha \cong v$. Therefore a possible difference in dispersion for protons passing through different pinholes is negligible and well below our resolution limit. It was not necessary to apply the electric field in order to separate the ions because of the ultra-short and high temporal contrast of the laser pulse, a pure proton beam was produced (Schnürer et al., 2007, Nickles et al., 2007).

A typical spectral image is shown in Fig.14a. As mentioned, this picture combines in principle the record of many independent spectrometers (in our case - 21) located under a different angle to the source and having the same dispersion constant and the bright spots in the right side of the figure are formed by energetic photons passing through pinholes and representing the axis of the spectrometers. The $\phi^{\circ}$ in the right side of Fig.14a are the tilt angles of the measured spectra from the spectrometers axes. The spectrum along the "central axis" has a 0o tilt. The spectrometers axes can be exactly defined with the measured 


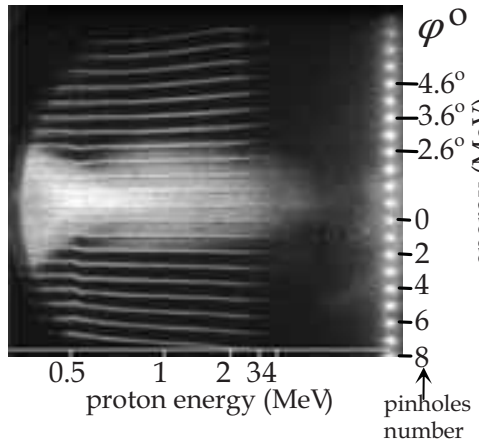

(a)

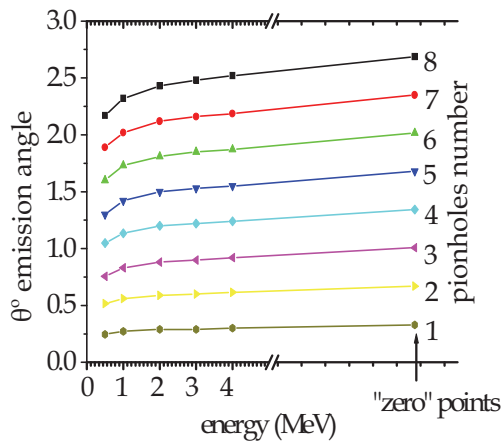

(c)

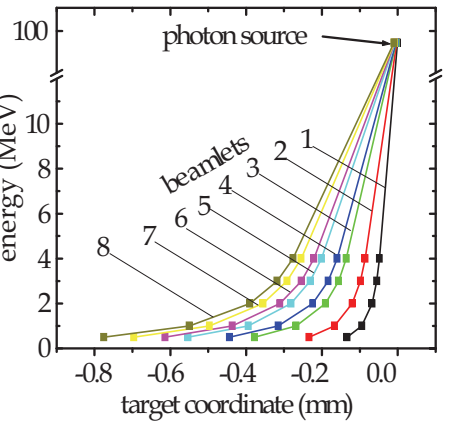

(b)

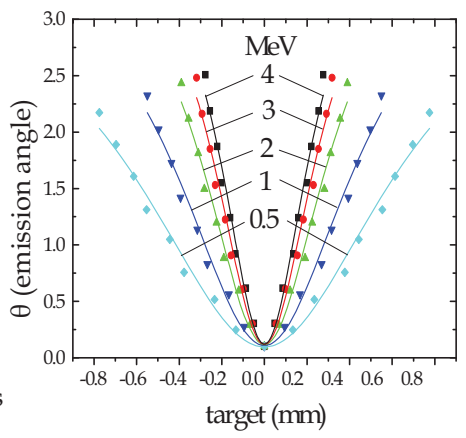

(d)

Fig. 14. (a) The imaged spectra through a row of pinholes are shown. Because the figure is symmetric to $\phi^{\mathrm{o}}=0$ line, on one half of the figure the dependence on the tilted angle $\phi^{\mathrm{o}}$ from axes is given (right upper side) and on the other half the numbering of the traces (right low side); (b) Energy of the emitted protons as function of target coordinate in the beamlets from 1st up to 8th is depicted; (c) Proton emission angle in each beam-let depending on proton energies; (d) emission angle of protons with $0.5,1,2,3,4 \mathrm{MeV}$ energies dependent on emission target coordinate.

energetic photons which are registered over a whole angle of $\pm 4.6^{\circ}$ from a source with an extension of about $20 \mu \mathrm{m}$. This means that initially the laser drives the hot electrons through the target with a divergence angle of about $45^{\circ}$. Having the axis of each pinholespectrometer and the angle of departure of the proton trace from this axis, the coordinate of the proton emission at the target surface and the emission angle can be calculated.

The striking features of Fig.14a are: (i) A clear spectral trace from each pinhole is observed. There are no crossing lines. This confirms that the beams of different energies are strongly laminar. (ii) The spectral traces are tilted from their axes. The "central" trace lies exactly on its spectrometer axis. Symmetrically, on both sides of the "central" trace all spectral traces have a tilt which increases with the distance to the central point with the angle $\phi^{\circ}$. Because of the good symmetry in the following figures we will show the spectra located only on one side from "central axes" in order to do not overload them. 
The analysis of the experimental data and the reconstruction of proton trajectories are straightforward. With the position of the pinholes along the linear array and the measured coordinates of the "zero points" both the target coordinate and the angle of the emitted protons could be calculated. This was done for different energies contributing to the spectral trace and by applying a simple geometrical relation. However, the scan of the magnetic field showed that the magnetic field lines are slightly curved in $x, y$ and $x, z$ planes and the existence of $\mathrm{B}_{\mathrm{y}}$ and $\mathrm{B}_{\mathrm{z}}$ components tilt the spectral traces. The protons will be deflected in $\pm \mathrm{x}$ direction due to both $\left(v_{z} \times B_{y}\right)$ and $\left(v_{y} \times B_{z}\right)$, where the $\mathrm{v}_{\mathrm{z}}$ and $\mathrm{v}_{\mathrm{y}}$ velocity component appears inside the magnet due to the dispersion. This was verified in a test experiment where a single proton beam was injected at ten different positions inside the magnet. The resulting instrument function was determined and used to unfold the measured raw data.

In Fig. $14 \mathrm{~b}$ the emitted proton energy is depicted as a function of the target coordinate derived from the $1^{\text {st }}$ up to the $8^{\text {th }}$ beam-let. It can be seen, that at the same target coordinate protons are emitted with different energies, while protons with the same energy arise from different target coordinates. As higher the energy of protons as smaller the region from where the emission occurs.

The proton emission coordinate as a function of the emitted proton energies (Fig.14b) of the $i^{\text {th }}$ beamlet can be fitted with an exponential function as:

$$
x_{E_{i}}=m_{i}=n_{i} \times \exp \left(-E_{i} / 0.93\right)
$$

where $m_{i}$ and $n_{i}$ are implicit functions of the target coordinates in relation to the pinhole $\mathrm{i}$; $m_{i}$ is the target coordinate from where the high energy protons have passed through the pinholes and therefore it is defined by the geometry of the pinhole array. It can be found that $m_{i}$ is a parabolic function of the period $(d)$ of the pinhole array: $m_{i}(\mathrm{~mm})=-0.018-0.038 d-0.003 d^{2}$, where $0.018 \mathrm{~mm}$ is the target coordinate from where the high energy protons have passed through the pinhole number zero.

Similarly, $n_{i}$ is also defined by the pinhole array but it shows how strong the target emission coordinate changes with the energy of the protons which are passing through a same pinhole. The $n_{i}$ is also a parabolic function of the pinhole array period: $n_{i}(m m)=-0.06-0.11 d-0.01 d^{2}$. Therefore, the relation between these two parameters is: $m_{i}=0.36 \times n_{i}$.

The proton trajectory in each beamlet depends on proton energy as shown in Fig.14c. The related emission angle of the protons is continuously changing with its kinetic energy. At the same angle only protons with different energies are emitted. Additionally, protons with low energy are emitted at a smaller angle compared to protons with high energy. The emission along the symmetry axis of the source is unique; the protons emitted under $0^{\circ}$ to the target normal are bearing any energy according to the spectral distribution.

The dependence of the proton emission angle on energy (Fig.14c) in different beamlets can be fitted with an exponential function as:

$$
\theta_{n, E_{i}}=\alpha_{n}-\beta_{n} \times \exp \left(-E_{i} / 0,93\right)
$$

where $\alpha_{n}$ is the emission angle of measured highest energy protons passing the $\mathrm{n}^{\text {th }}$ pinhole. The $\alpha_{n}$ is a linear function from the pinhole array period: $\alpha_{n}=-0,02+0.65 d$, showing a linear increase of the emission angle of high energy protons with the target coordinate and $0.02^{\circ}$ is the opening angle of the beam which is passing through the pinhole number 0 
(Fig.13). The parameter $\beta_{n}$ shows how strong the emission angle of protons changes if they are passing through the same pinhole with different energies. The $\beta_{n}$ is the parabolic function of the pinhole array period $(d)$ and can be expressed as $\beta_{n}=-0.04-0.07 d+0.005 d^{2}$. It means that going with increasing distance to the centre and from one pinhole to the next one the angle increases in respect to a similar change of the energy.

The emission angles of protons with the same energy but with different emission coordinates in relation to the 16 beamlets which are symmetrically located on both sides of the "central axes" are shown in Fig.14d. For each proton energy the data can be fitted with the Lorentz function and it can be found that the width of the source $(\Delta x)$ is an exponential function of the proton energies: $\Delta x \sim E^{-0.5}$.

With these relations one can reconstruct the proton trajectories as they are emerging from the target. One can see in Fig.15a, where as an example 0.5 and $4 \mathrm{MeV}$ proton trajectories are shown, that protons with a same energy are accelerated as an expanding laminar beam. The emission angle increases with the target coordinate (or the distance from the source centre) and higher energies belong to a large emission angle if the same target region is analyzed. Additionally, the measured high energy protons are emitted from a smaller target region than the lower energetic once.
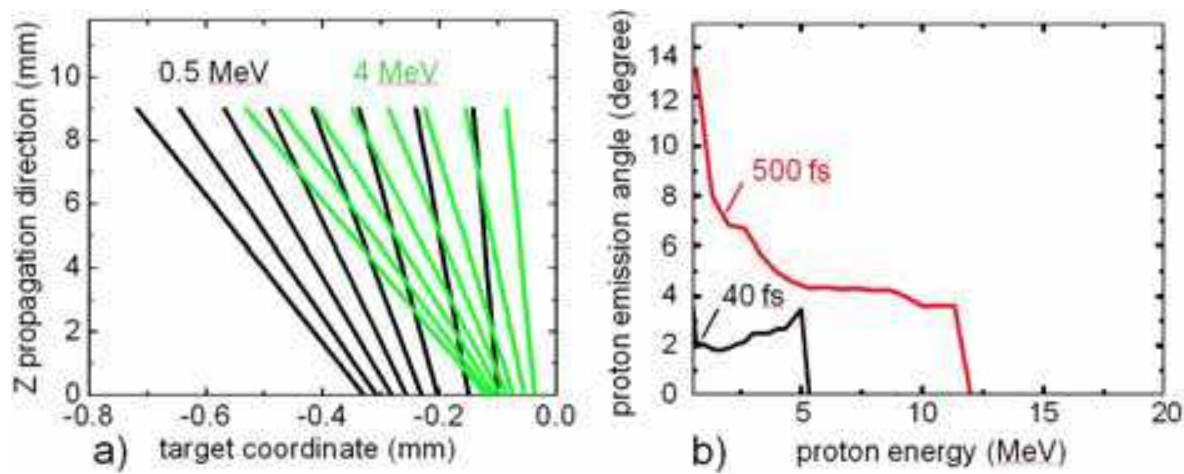

Fig. 15. a) Reconstructed proton trajectories as they are emerging from the target. As an example are shown 0.5 and $4 \mathrm{MeV}$ proton trajectories. b) Simulated angular spread of proton emission depending on proton energy for $40 \mathrm{fs}$ and $500 \mathrm{fs}$ laser pulses.

The discussion above makes clear, that each beam-let is formed by an unique combination of energy, target coordinate and emission angle of the protons. The low energy protons are emitted with a smaller angle than the high energetic ones (Fig.15a). At first glance this observation seems to be in contradiction with a statement in (Cowan et al., 2004) that the angular envelope of the protons with increasing proton energy is decreasing. There are two things which make a decisive comparison to other experiments and its conclusions difficult. First, there are no data known to us from the literature with a similar resolution in order to make a direct comparison concerning the emission of the central part of the beam as we performed. Second, due to the present limitation of the observed range of angles we did not look to the maximum extension of the source as a function of energy. The latter one is in other works mainly attributed to the source size. If one calculates the ratio between the emission coordinates (source size) and the emission angle as a function of the ion kinetic energy one finds a qualitative similar functional dependence of our data and those from 
other work. Nevertheless a final conclusion for different experimental parameters realized in different work should be drawn from data with similar resolution. However, the discussed features unambiguously follow directly from the experimental findings. Also recent experiments showed (Roth et al., 2005) that the highest energy protons are emitted in the central, high density portion of the sheath distribution, which agrees with the present results (Fig.14b). Nevertheless, it is likely that both the used long pulse (350 - $850 \mathrm{ps)}$ and high laser energy (20 - $30 \mathrm{~J}$ ) (Cowan et al., 2004), in contrast to our ultra-short pulse (40 fs) with "low" energy $(0.7 \mathrm{~J})$, and more importantly the high temporal laser pulse contrast $\left(10^{-7}\right.$ - 10-8) (Nickles et al., 2007) are the decisive parameters for the proton source formation and emission characteristics of the accelerated particles.

In order to look particularly to proton acceleration scenarios in case of long and high energy laser pulses in comparison to short and low energy laser pulses 2D particle-in-cell (PIC) simulations were performed. The simulation box had a size of $150 \mu \mathrm{m} \times 150 \mu \mathrm{m}$. The $10 \mu \mathrm{m}$ thick and $150 \mu \mathrm{m}$ long target was located at the coordinate $12.0<x(\mu \mathrm{m})<22.0$. The preplasma was set $0.5 \mu \mathrm{m}$ in front of the target and composed of $\mathrm{Al}^{3+}$ cold ions and $500 \mathrm{eV}$ electrons with a density $20 \mathrm{n}_{\mathrm{c}}$. The $\mathrm{n}_{\mathrm{c}}$ denotes the critical density. A proton contamination layer was buried within a distance of $0.5 \mu \mathrm{m}$ from the rear surface. The laser with a wavelength of $0.8 \mu \mathrm{m}$ and focused to $10 \mu \mathrm{m}$ was irradiating the target under $0^{\circ}$. The laser pulse durations were set as $40 \mathrm{fs}$ and $500 \mathrm{fs}$ and had Gaussian profiles both in space and time. The electromagnetic field was damped at the boundary of the simulation box, and the particles were reflected with thermal energy.

The simulation results of the proton energy dependence on the emission angle are shown in Fig.15b. The full simulation time was $900 \mathrm{fs}$ for the short, and $1400 \mathrm{fs}$ for the long laser pulse. The difference is apparent: For the 500 fs laser pulse irradiation the angular spread of the accelerated protons is gradually increasing with decreasing proton energies, as it was observed in (Cowan et al., 2004). This is in contrast to the case of the $40 \mathrm{fs}$ laser pulse irradiation where the higher energetic protons have the largest angular spread, and the emission angle decreases with the proton energy. In the long pulse case, the sheath field accelerates protons for roughly $500 \mathrm{fs}$, where the acceleration is described by hydrodynamic plasma evolution (Mora, 2003). In short pulse case the maximum sheath field is sustained only about $40 \mathrm{fs}$ while protons are about to be accelerated. When the proton acceleration starts, the sheath field is still expanding and the accelerating front has a large curvature, which results in larger angular spread for higher energetic protons. The simulated energies and the angular spreads are quite comparable to that presented here and to the experimental results in (Cowan et al., 2004).

Additionally, results of simulation for the angular behaviour of protons emitted from different target coordinates are similar to those shown in Fig. $15 \mathrm{~b}$ for both $40 \mathrm{fs}$ and $500 \mathrm{fs}$ laser irradiation cases. Therefore, one can conclude, that the observed features in the present experiments is an unique property of the ultrashort and high contrast laser pulse acceleration scenario.

On base of the found interconnections between the target coordinate, emission angle and energy of emitted protons, one can constitute the following rules:

- The proton emission is directed to the target normal and it is symmetric to the laser axis.

- Protons with different energies are emitted such that: as higher the proton energy as smaller the source size but as bigger the emission angle in relation to the source size 
- Protons with the same energy are emitted from different target coordinates such that: as far away from the centre as larger the emission angle

- Protons which are emitted from the same target coordinate but with different energies obey to the following: as higher the energy as larger the emission angle.

\subsection{Correlation of spectral, spatial and angular characteristics of proton source}

The measurements discussed in section I. 4 were made around the central symmetry axis of the source. Due to the limited size of the detector only protons with 0.5 to $4 \mathrm{MeV}$ energies emitted within a 2-3 degree angle were analysed. In order to acquire an understanding about the whole source behaviour, broader proton energy ranges have to be covered. Additionally, it is of interest and necessary to explore to what extent the measured proton source properties near the central symmetry axis are applicable to the whole and much more extended proton source. Particularly in view of planning proton beam steering systems it is necessary to know up to which point the measured energy dependence of the angular emission characteristic is preserved.

Here, the investigation of the source emission characteristics was extended at larger angles from the central symmetry axis and in a rather large range of proton kinetic energies from $0.06 \mathrm{MeV}$ up to $4 \mathrm{MeV}$.

The measurement of the proton emission spectrum was carried out similar to section 3.1 Being restricted by the size of the detector a good compromise between the requirements of high magnification and of an extended spectral range was found by locating the pinhole array at a distance of about $\mathrm{a}=30 \mathrm{~mm}$ from the target surface and the detector screen at $\mathrm{b}=$ 195, c $=255 \mathrm{~mm}$ from the pinholes (Fig.13). The inset of Fig.13 shows the imaging geometry which allowed a complete characterisation of the source in the whole spectral range.

In our previous section 3.1 the pinhole array was aligned in such a way that the central symmetry axis of the plasma crossed the central pinhole. In the measurements reported here we applied an imaging geometry with higher magnification and, distinctively, the pinhole array was shifted along the $\mathrm{Y}$ axis (i.e. parallel to the target surface and away from the ion beam central axis) (see inset of Fig.13). This shift, of about $2 \mathrm{~mm}$, gave a corresponding shift of about $40 \mathrm{~mm}$ for the positions of the "zero" points on the detector. The observation angle extended up to 4.77 degrees relative to central symmetry axis of the plasma. In fact, with this geometry we imaged a source area centred at about $70 \mu \mathrm{m}$ from the source centre.

The respective spectral traces cover the high energy part of the spectra (Fig.16a) in the same way as in section 3.1 but extend the source imaging region to cover energies down to 0.2 $\mathrm{MeV}$ protons. Fig.16a looks very similar to Fig.14a in section 3.1 where a lower magnification was applied. The data analysis confirmed that the observed spatial and angular characteristics of the emitted protons are exactly the same as in the case of axissymmetric detection (section 3.1) and can be treated in a similar manner. The tilts of the spectra from their axes are still symmetric relative to the zero degree trace (i.e. the trace due to the pinhole on-axis with the interaction point). For the $45^{\circ}$ incidence on target as employed in the experiment the focus is elliptically shaped and has two main symmetry axes and therefore one should expect the source area to show also this elliptical symmetry. We have measured along the short main axis of the elliptically shaped source area. As a result also the tilts of the trajectories for the corresponding energies from the main axis are symmetric relative to the "central" trace. The data analysis was carried out by treating the signal observed as the result of 21 independent spectrometers as defined by the pinholes. 


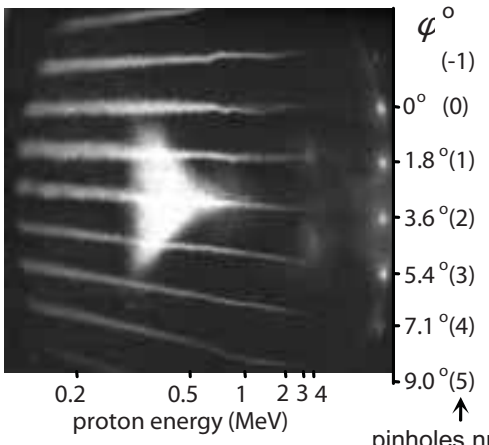

(a)

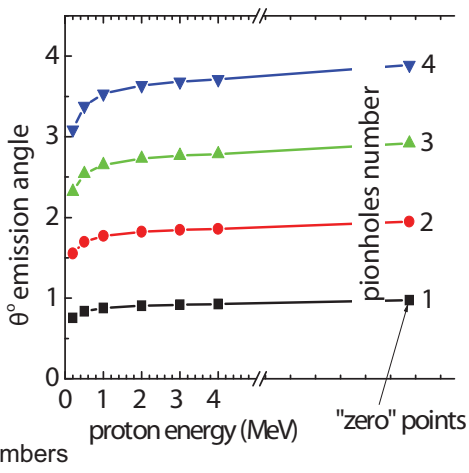

(b)

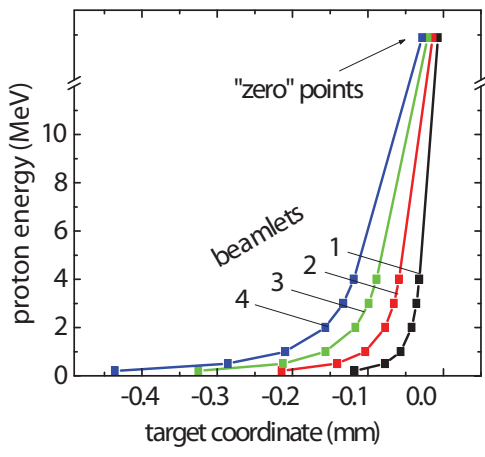

(c)

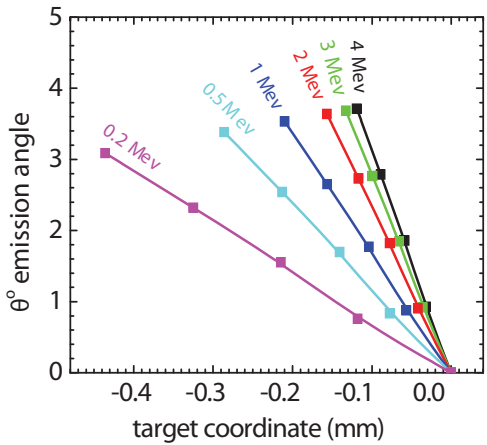

(d)

Fig. 16. a) Spectral traces of a row of pinhole images: On the right side of the figure the tilt angle $\phi^{\circ}$ of the spectral traces from the central symmetry axes corresponding to $\phi^{\circ}=0$ line (target normal) and respective numbers of beam-lets (in brackets) are given. b) Proton emission angle in each beam-let depending on proton energies in beamlets from 1st up to 4th is depicted. c) Emitted proton energy as function of target coordinates in beamlets from 1 st up to 4th. d) Emission angle of protons with $0.2,0.5,1,2,3$, and $4 \mathrm{MeV}$ energies dependent on target emission coordinate is exhibited.

Each spectrometer axis was defined by the coordinate of the "zero" point and the coordinate of the corresponding pinhole (Fig.13). If protons within the observed energy range are all emitted from the same source point the projection of their spectrum on the X-axis of the detector (Fig.13) should be a point, i.e. the line describing the spectrum should be along the $Y$ axis. The measurement showed instead that the lines described by the spectra in the detector plane are tilted with respect to the $\mathrm{Y}$ axis i.e. the projection on the $\mathrm{X}$ axis is different for different energies (Fig.16a). From the $\mathrm{X}$ coordinate of the projection for a particular energy the corresponding pinhole coordinate and assuming straight propagation of the particles, the coordinate on the target surface from where the corresponding proton is emitted (inset of Fig.13) can be calculated.

The "zero" points coupled with the position of the corresponding pinhole, are used as a reference to define the axes of the spectrometers. When using MCP detectors, the "zero" 
points can be due to X-rays (Ter-Avetisyan et al., 2009b) and neutral particles (Busch, et al., 2003, McKenna et al., 2007) which are undeflected by the magnetic field in the spectrometers.

The data analysis shows that the trend observed in section 3.1 of the proton emission angle decreasing with decreasing proton energy is preserved in these measurements (Fig.16b). Additionally, higher energy protons are emitted from a smaller target region (Fig.16c) with higher divergence (Fig.16d) while the proton source for lower energies becomes larger and has a lower divergence. These trends confirm the assumption that "each source point behaves similarly" which allows one to characterise the general source behaviour independently from the specific area of the source under observation. Therefore a similar approach for the calculation of the proton emission trajectories (section 3.1) has been applied.
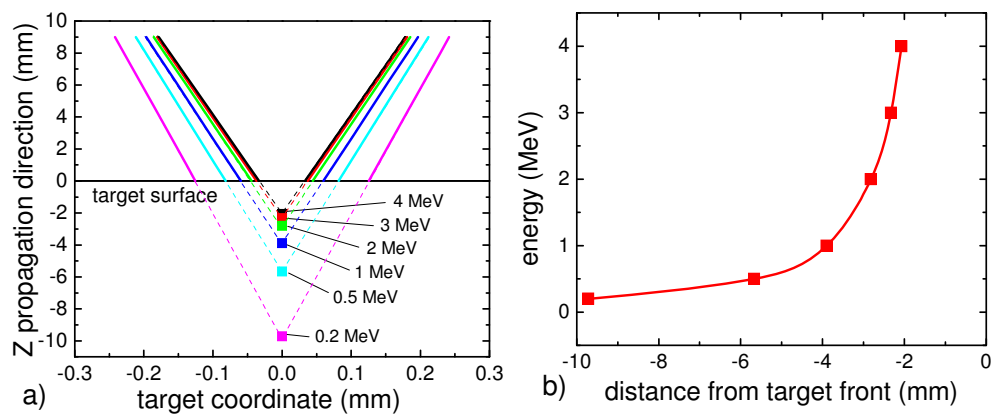

Fig. 17. Reconstructed proton trajectories as they are emerging from the target. As an example a) proton trajectory passing through 1 and -1 pinholes are shown. The virtual source position in front of the target is deduced by linear interpolation of the proton trajectory lines (dashed lines). b) The virtual source position in front of the target for different proton energies is shown.

The trajectories of protons with different energies, which pass for example through the pinholes +1 and -1 are deduced from the spectral traces and shown in Fig.17a. One can adopt a virtual source concept to describe the behaviour of a laminar ion source, as discussed in (Borghesi, et al., 2004). If one assumes that the propagation of the proton beam after the acceleration process is ballistic and there is no ion interaction within the beam, the proton trajectories are straight lines (inset of Fig.13) and one can trace back these trajectories to a virtual source $\left(\mathrm{VS}_{\mathrm{p}}\right)$ in front of the target as shown in Fig.17a (dash lines).

The virtual source position in front of the target changes for different proton energies (Fig.17b), which means that the protons with different energies are emitted with a different curvature of the accelerating sheath field. In the inset of Fig.13 the virtual source points for protons with different energies, where $E_{p 1}>E_{p 2}$, are shown. This hints at a change of the sheath field curvature during the acceleration process.

As a next step measurements with a larger displacement of the observation axis were realized (i.e. the pinhole array was shifted sideways so that the central pinhole was further away from the interaction axis). This set of measurements was aimed to explore the emission characteristics far from the source centre. Furthermore, the measurement was extended to protons with even lower energy. A displacement of the observation axis of about $1 \mathrm{~mm}$ from the central source axis was set, which corresponds (for the central pinhole 
of the array) to an observation angle of about 4.88 degree relative to the central symmetry axis of the plasma. The measured proton spectra are shown in Fig.18a.

Due to the limited size of the detector the "zero points" are not visible in this case. As the "zero point" coordinates are needed for the trajectory reconstruction now the deflecting electric field of the Thomson spectrometer was switched on in separate shots to perform an energy calibration by using deflection of protons in an applied electric field. From the parabolic traces the corresponding "zero points" were extrapolated and these extrapolated coordinates have been used to analyze the measurements for the respective pinhole and spectrometer setting. The proton emission angle depends on proton energies as shown in Fig.18b. The data show clearly (Fig.18b) the continuous decrease of the proton emission angles relative to target normal with decreasing proton energies. This tendency holds down to proton energies of about $0.14 \mathrm{MeV}$ which are emitted along the target normal; therefore one can say that they are "collimated". The proton beam at energies lower than $0.14 \mathrm{MeV}$ is
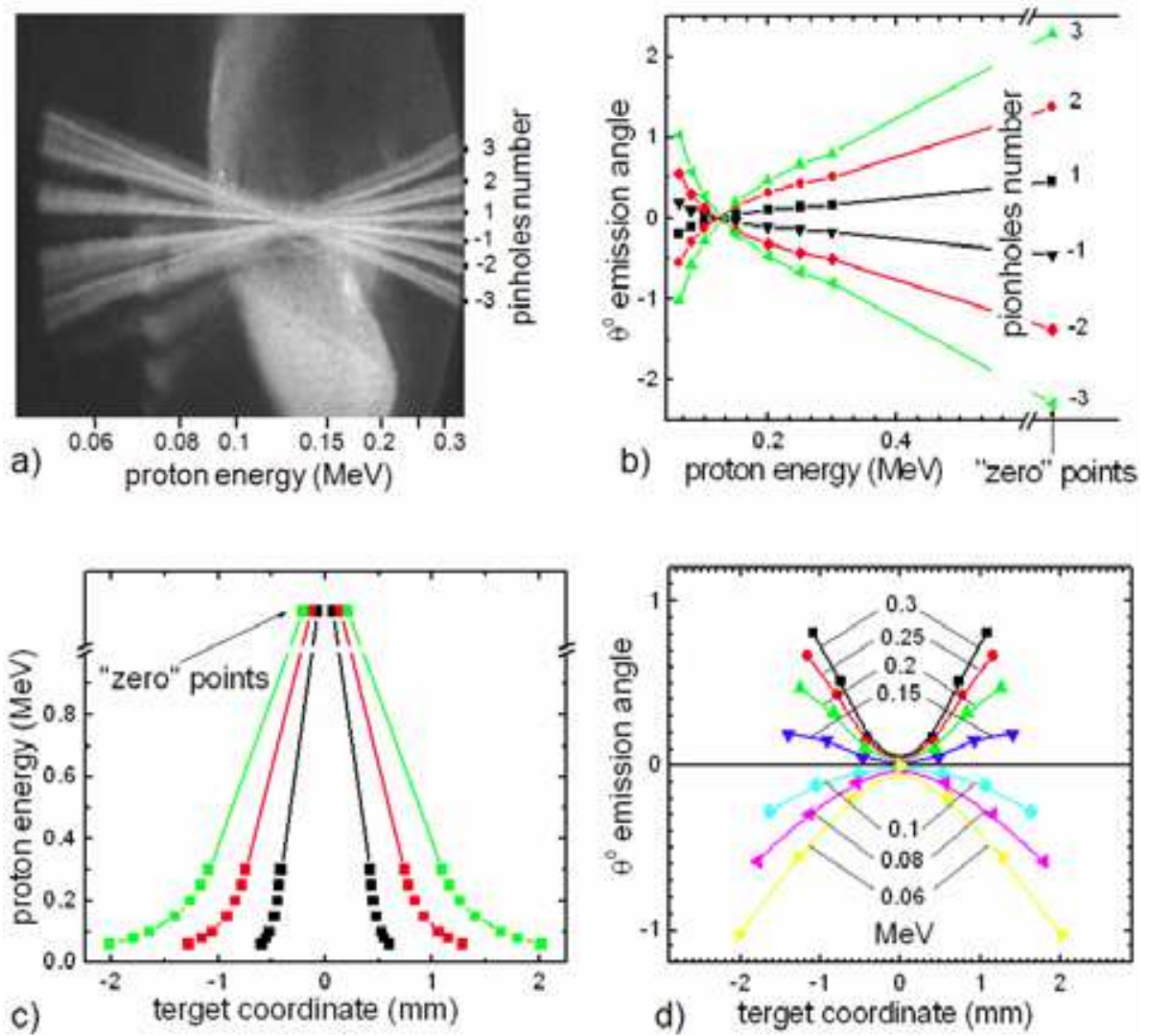

Fig. 18. a) The imaged spectra through a row of pinholes are shown. On the right side the numbers of beam-lets are given. b) Proton emission angle in each beam-let depending on proton energies in beam-lets is depicted. c) Emitted proton energy as function of target coordinate and (d) emission angle of protons with $0.06,0.08,0.1,0.15,0.2,0.25$ and $0.3 \mathrm{MeV}$ energies dependent on emission coordinate is exhibited. $\theta^{0}=0$ is the angle of target normal. 
convergent and in the detector plane one can see the crossing of trajectories at about 0.12 $\mathrm{MeV}$ (Fig.18b). For such low proton energies the source size becomes as large as a few $\mathrm{mm}$ (Fig.18c). Concerning this largely extended source area we thus found that the beam becomes converging (Fig.18d) as much as the proton energy decreases. On basis of this measurement geometry the proton trajectories are calculated and traced back to the target surface (cf. Fig.19a).

To our knowledge this is the first observation showing that a proton beam driven by an ultra-short laser pulse consists of a divergent, a "collimated" and a converging component, with the divergence varying continuously as a function of energy.

The structure of the transverse electric field obtained in 2D PIC simulations performed at the conditions as in section 3.1 gave some qualitative indication of possible reasons for the observed divergence-to-convergence behaviour of the proton beam. The simulation starts at $\mathrm{t}=0$ and the laser peak reaches the target surface at $\mathrm{t}=120 \mathrm{fs}$. The structure of the transverse electric field is plotted in Fig.19b. The bell-shaped red lines (4 are visible) represent isodensity contour plots of protons in the accelerated bunch.

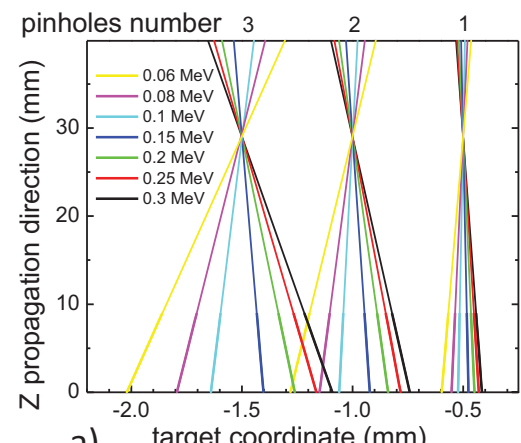

a) target coordinate $(\mathrm{mm})$

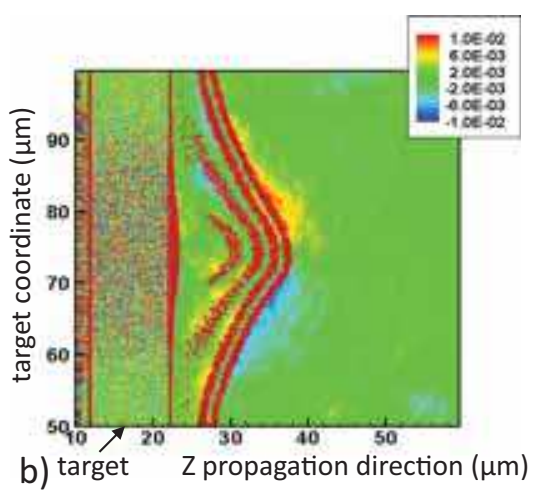

b) target $\quad \mathrm{Z}$ propagation direction $(\mu \mathrm{m})$

Fig. 19. a)Reconstructed proton trajectories as they are emerging from the target with the energies $0.06,0.08,0.1,0.15,0.2,0.25$ and $0.3 \mathrm{MeV}$ and passing through the 1 st, 2 nd, and 3rd pinholes are shown. b) 2D PIC simulation results: transverse component of sheath field at $t=500 \mathrm{fs}$ (the parameters of the simulation are explained in the text).

The protons propagate from the left to the right. At the ion front we observe transverse field components which are opposite with respect to the $y=75$ symmetry line: the field is directed up for $y>75$ (red colour code) and down for $y<75$ (colour code blue). The transverse field is most intense at the acceleration front which causes radial deflection and angular spreading of the higher energy protons. Behind the ion front we observe weaker field strength and finally a change in the field polarity (at $x<25)$ : For the region $y>75$ we observe now light blue coded areas (i.e. field directed down) and for $\mathrm{y}<75$ yellow ones (field directed up), respectively. Thus the slower protons, which are behind the fast ion front, experience a transverse field component which can in principle result in converging trajectories, as observed in the experiment for rather low energetic protons.

The situation might be different in the case of ions accelerated by long (ps) laser pulses, where the longitudinal momentum gained by the ions is higher and transverse fields may be less effective in spreading the high energy ions. 
Summarizing the emission properties of a proton beam accelerated by a high contrast ultrashort laser pulse have to be understood as follows. A target source point emits protons within a broad range of energies. Taken two energies $\mathrm{E}_{1}$ and $\mathrm{E}_{2}$ such that $E_{2}<E_{1}$, the relation of the proton emission angles is $\theta_{E_{1}}<\theta_{E_{1}}$. Any point located at a distance $\mathrm{d}$ from the target rear surface and located within the total beam cone will be crossed by the trajectories of ions with different energies which are stemming from different target-source coordinates. This means, only a certain target area $(S)$ is contributing to the ion spectrum which is measured at some point in the beam cone and at a certain distance from the target rear surface. As a result, the spectrum of the protons in that point can be controlled by changing the proton source area. It might be that our results apply also to the results of Ref. Schwoerer et al., (2006), where a proton beam with a small energy spread was detected when the proton source area was restricted.

The data also indicates that the proton beam is emitted from the target so that for high energies it is divergent and becomes convergent for low energies (below $0.2 \mathrm{MeV}$ ). 2D PIC simulations provide indications on how the effect of field components transverse to the beam propagation axis could lead to these features. It will be interesting to test in future experiments how this behavior will scale if the cut-off energies in the ion beam are significantly enhanced using a laser driver providing much higher energy and intensity.

Additionally comparative investigations with longer driver pulses may clarify whether (as it is likely) the divergence behavior measured here is an exclusive feature of short pulse interactions.

\section{Conclusion}

In this article recent studies of phenomena in relativistic proton acceleration have been reviewed. Experimental results have shown unique properties of protons accelerated by ultrashort laser pulses, which opens prospects for a broad range of applications (Borghesi et al., 2006).

Significant advances in laser technology are likely to expect for the next years, leading to further enhancement of both the intensity of the extremely short pulses and the average power. These developments will surely stimulate the elaboration of new ideas and more advanced diagnostic developments for measuring the effects not even thought of today. All of this will open up new research areas, pushing current activities to new frontiers, and generate further excitement in the field of laser-matter physics.

\section{References}

Allen, M., Sentoku, Y., Audebert, P., Blazevic, A., Cowan, T., et al., (2003). Proton spectra from ultraintense laser-plasma interaction with thin foils: Experiments, theory, and simulation. Phys Plasmas 10, 3283-3289.

Beg, F.N., Bell, A.R., Dangor, A.E., Danson, C.N., Fews, A.P., et al., (1997). A study of picosecond laser-solid interactions up to $10^{19} \mathrm{~W} \mathrm{~cm}^{-2}$. Phys Plasmas 4, 447-457.

Blochin, M.A., (1957). Physik der Röntgenstrahlen. Akademischer Verlag, Berlin, pp. 70.

Borghesi, M., Fuchs, J., Bulanov, S.V., Mackinnon, A.J., Patel, P., \& Roth, M. (2006). Fast ion generation by high intensity laser-irradiation of foils and applications. Fus. Sci Tech 49, 412-439.

Borghesi, M., Mackinnon, A.J., Campbell, D.H., Hicks, D.G., et al., (2004). Multi-MeV proton source investigations in ultraintense laser-foil interactions. Phys Rev Lett 92, 55003. 
Busch, S., Schnurer, M., Kalashnikov, M., Schonnagel, H., Stiel, H., et al., (2003). Ion acceleration with ultrafast lasers. Appl Phys Lett 82, 3354-3356.

Clark, E.L., Krushelnick, K., Davies, J.R., Zepf, M., Tatarakis, et al., (2000a). Measurements of energetic proton transport through magnetized plasma from intense laser interactions with solids. Phys Rev Lett 84, 670-673.

Clark, E.L., Krushelnick, K., Zepf, M., Beg, F.N., Tatarakis, M., et al., (2000b). Energetic heavy ion and proton generation from ultraintense laser-plasma interactions with solids. Phys Rev Lett 85, 1654-1657.

Cowan, T.E., Fuchs, J., Ruhl, H., Kemp, A., Audebert, P., et al., (2004). Ultralow emittance, multi-MeV proton beams from a laser virtual-cathode plasma accelerator. Phys Rev Lett 92, 204801.

Denavit, J., (1992). Absorption of high-intensity subpicosecond lasers on solid density targets. Phys Rev Lett 69, 3052-3055.

Estabrook, K., \& Kruer, W.L., (1978). Properties of resonantly heated electron distributions. Phys Rev Lett 40, 42-45.

Fews, A.P., Norreys, P.A., Beg, F.N., Bell, A.R., Dangor, A.E., et al., (1994). Plasma Ion emission from high-intensity picosecond laser-pulse interactions with solid targets. Phys Rev Lett 73, 1801-1804.

Forslund, D.W., \& Brackbill, J.U., (1982). Magnetic-field-induced surface transport on laserirradiated foils. Phys Rev Lett 48, 1614-1617.

Fuchs, J., Cowan, T.E., Audebert, P., Ruhl, H., Gremillet, L., et al., (2003). Spatial uniformity of laser-accelerated ultrahigh-current $\mathrm{MeV}$ electron propagation in metals and insulators. Phys Rev Lett 91, 255002.

Gitomer, S.J., Jones, R.D., Begay, F., Ehler, A.W., Kephart, J.F., \& Kristal, R., (1986). Fast ions and hot-electrons in the laser-plasma interaction. Phys Fluids 29, 2679-2688.

Griem, H.R., (1964). Plasma Spectroscopy. McGraw-Hill, New York, pp. 113.

Hegelich, M., Karsch, S., Pretzler, G., Habs, D., Witte, et al., (2002). MeV ion jets from shortpulse-laser interaction with thin foils. Phys Rev Lett 89, 085002.

Hemberg, O., Hansson, B.A.M., Berglund, M., \& Hertz, H.M., 2000. Stability of droplettarget laser-plasma soft x-ray sources. Journal of Applied Physics 88, 5421-5425.

Kalachnikov, M.P., Karpov, V., Schonnagel, H., \& Sandner, W., (2002). 100-terawatt titanium-sapphire laser system. Laser Physics 12, 368-374.

Kaluza, M., Schreiber, J., Santala, M.I.K., Tsakiris, G.D., et al., (2004). Influence of the laser prepulse on proton acceleration in thin-foil experiments. Phys Rev Lett 93, 045003.

Karsch, S., Dusterer, S., Schwoerer, H., Ewald, F., Habs, D., et al., (2003). High-intensity laser induced ion acceleration from heavy-water droplets. Phys Rev Lett 91, 015001.

Kishimoto, Y., Mima, K., Watanabe, T., \& Nishikawa, K., (1983). Analysis of fast-ion velocity distributions in laser plasmas with a truncated maxwellian velocity distribution of hot-electrons. Physics of Fluids 26, 2308-2315.

Kruer, W.L., \& Estabrook, K., (1985). Jxb Heating by very intense laser-light. Physics of Fluids 28, 430-432.

Lefebvre, E., \& Bonnaud, G., (1997). Nonlinear electron heating in ultrahigh-intensity-laser plasma interaction. Phys Rev E 55, 1011-1014.

Lindau, F., Lundh, O., Persson, A., McKenna, P., Osvay, K., et al., (2005). Laser-accelerated protons with energy-dependent beam direction. Phys Rev Lett 95, 175002.

Mackinnon, A.J., Sentoku, Y., Patel, P.K., Price, D.W., Hatchett, S., et al., (2002). Enhancement of proton acceleration by hot-electron recirculation in thin foils irradiated by ultraintense laser pulses. Phys Rev Lett 88, 215006. 
Mackinnon, A.J., Borghesi, M., Hatchett, S., Key, M.H., Patel, P.K., et al., (2001). Effect of plasma scale length on multi-MeV proton production by intense laser pulses. Phys Rev Lett 86, 1769-1772.

Maksimchuk, A., Gu, S., Flippo, K., Umstadter, D., \& Bychenkov, V.Y., (2000). Forward ion acceleration in thin films driven by a high-intensity laser. Phys Rev Lett 84,4108 4111.

Malka, G., \& Miquel, J.L., (1996). Experimental confirmation of ponderomotive-force electrons produced by an ultrarelativistic laser pulse on a solid target. Phys Rev Lett 77, 75-78.

Manclossi, M., Santos, J.J., Batani, D., Faure, J., Debayle, A., et al., (2006). Study of ultraintense laser-produced fast-electron propagation and filamentation in insulator and metal foil targets by optical emission diagnostics. Phys Rev Lett 96, 125002.

McKenna, P., Carroll, D.C., Clarke, R.J., Evans, R.G., Ledingham, K.W.D., et al., (2007). Lateral electron transport in high-intensity laser-irradiated foils diagnosed by ion emission. Phys Rev Lett 98, 145001.

Mora, P., (2003). Plasma expansion into a vacuum. Phys Rev Lett 90, 185002.

Nakamura, T., Kato, S., Nagatomo, H., \& Mima, K., (2004). Surface-magnetic-field and fastelectron current-layer formation by ultraintense laser irradiation. Phys Rev Lett 93, 265002.

Nickles, P.V., Ter-Avetisyan, S., Schnuerer, M., Sokollik, T., Sandner, W., et al., (2007). Review of ultrafast ion acceleration experiments in laser plasma at Max Born Institute. Laser Part Beams 25, 347-363.

Nishihara, K., Amitani,H., Murakami,M., Bulanov, S.V. \& Esirkepov, T.Zh., (2001). High energy ions generated by laser driven Coulomb explosion of cluster. Nucl Instrum and Meth A 464, 5 .

Passoni, M., Tikhonchuk, V.T., Lontano, M., \& Bychenkov, V.Y., (2004). Charge separation effects in solid targets and ion acceleration with a two-temperature electron distribution. Phys Rev E 69, 026411.

Pukhov, A., Sheng, Z.M., \& Meyer-ter-Vehn, J., (1999). Particle acceleration in relativistic laser channels. Phys Plasmas 6, 2847-2854.

Pukhov, A., (2001). Three-dimensional simulations of ion acceleration from a foil irradiated by a short-pulse laser. Phys Rev Lett 86, 3562-3565.

Roth, M., Brambrink, E., Audebert, P. et al., (2005). The generation of high-quality, intense ion beams by ultra-intense lasers. Plasma Phys Control Fusion 47, B841-B850.

Schnurer, M., Ter-Avetisyan, S., Nickles, P.V., \& Andreev, A.A., (2007). Influence of target system on the charge state, number, and spectral shape of ion beams accelerated by femtosecond high-intensity laser pulses. Phys Plasmas 14, 033101.

Schreiber, J., Ter-Avetisyan, S., Risse, E., Kalachnikov, M.P., Nickles, P.V.,et al., (2006). Pointing of laser accelerated proton beams. Phys Plasmas 13, 033111.

Schwoerer, H., Pfotenhauer, S., Jackel, O., Amthor, K.U., Liesfeld, B., et al., (2006). Laserplasma acceleration of quasi-monoenergetic protons from microstructured targets. Nature 439, 445-448.

Shorokhov, O., \& Pukhov, A., (2004). Ion acceleration in overdense plasma by short laser pulse. Laser Part Beams 22, 175-181.

Silva, L.O., Marti, M., Davies, J.R., Fonseca, R.A., Ren, C., Tsung, F.S., \& Mori, W.B., (2004). Proton shock acceleration in laser-plasma interactions. Phys Rev Lett 92, 015002.

Snavely, R.A., Key, M.H., Hatchett, S.P., Cowan, T.E., et al., (2000). Intense high- energy proton beams from petawatt-laser irradiation of solids. Phys Rev Lett 85, 2945-2948. 
Sokollik, T., Schnurer, M., Ter-Avetisyan, S., et al., (2008). Transient electric fields in laser plasmas observed by proton streak deflectometry. Appl Phys Lett 92, 091503.

Stein, J., Fill, E., Habs, D., Pretzler, G., \& Witte, K., (2004). Hot electron diagnostics using Xrays and Čerenkov radiation. Laser Part Beams 22, 315-321.

Tajima, T., \& Dawson, J.M., (1979). Laser Electron-Accelerator. Phys Rev Lett 43, 267-270.

Ter-Avetisyan, S., Schnurer, M., Stiel, H., \& Nickles, P.V., (2003). A high-density sub-micron liquid spray for laser driven radiation sources. J Phys D: Appl Phys 36, 2421-2426.

Ter-Avetisyan, S., Schnurer, M., Busch, S., Risse, E., et al., (2004a). Spectral dips in ion emission emerging from ultrashort laser-driven plasmas. Phys Rev Lett 93, 155006.

Ter-Avetisyan, S., Schnürer, M., Busch, S., \& Nickles, P.V., (2004b). Negative ions from liquid microdroplets irratiated with ultrashort and intense laser pulses. J Phys B: At Mol Opt Phys 37, 3633 - 3640.

Ter-Avetisyan, S., Schnurer, M., \& Nickles, P.V., (2005). Time resolved corpuscular diagnostics of plasmas produced with high-intensity femtosecond laser pulses.J Phys D: Appl Phys 38, 863-867.

Ter-Avetisyan, S., Schnurer, M., Nickles, P.V., Sokollik, T., Risse, E., et al., (2008). The Thomson deflectometer: A novel use of the Thomson spectrometer as a transient field and plasma diagnostic. Rev Sci Instrum 79, 033303.

Ter-Avetisyan, S., Schnurer, M., Nickles, P.V., Sandner, W., Nakamura, T., \& Mima, K., (2009a). Correlation of spectral, spatial, and angular characteristics of an ultrashort laser driven proton source. Phys Plasmas 16, 043108.

Ter-Avetisyan, S., Ramakrishna, B., Doria, D., Sarri, G., Zepf, M., et al., (2009b). Complementary ion and extreme ultra-violet spectrometer for laser-plasma diagnosis. Rev Sci Instrum 80, 103302.

Tikhonchuk, V.T., (2002). Interaction of a beam of fast electrons with solids. Phys Plasmas 9, 1416-1421.

Wei, M.S., Mangles, S.P.D., Najmudin, Z., Walton, B., Gopal, A., et al., (2004). Ion acceleration by collisionless shocks in high-intensity-laser-underdenseplasmainteraction. Phys Rev Lett 93, 155003.

Weibel, E.S., (1959). Spontaneously growing transverse waves in a plasma due to an anisotropic velocity distribution. Phys Rev Lett 2, 83-84.

Wickens, L.M., Allen, J.E., \& Rumsby, P.T., (1978). Ion emission from laser-produced plasmas with 2 electron temperatures. Phys Rev Lett 41, 243-246.

Wickens, L.M., \& Allen, J.E., (1979). Free expansion of a plasma with 2 electron temperatures. J Plasma Phys 22, 167-185.

Wickens, L.M., \& Allen, J.E., (1981). Ion emission from laser-produced, multi-ion species, 2electron temperature plasmas. Phys Fluids 24, 1894-1899.

Wilks, S.C., Kruer, W.L., Tabak, M., \& Langdon, A.B., (1992). Absorption of ultra-intense laser-pulses. Phys Rev Lett 69, 1383-1386.

Wilks, S.C., Langdon, A.B., Cowan, T.E., Roth, M., Singh, M., et al., (2001). Energetic proton generation in ultra-intense laser-solid interactions. Phys Plasmas 8, 542-549.

Zepf, M., Clark, E.L., Beg, F.N., Clarke, R.J., Dangor, A.E., et al., (2003). Proton acceleration from high-intensity laser interactions with thin foil targets. Phys Rev Lett 90, 064802

Zhidkov, A.G., Sasaki, A., Fukumoto, I., Tajima, T., Auguste, T., et al., (2001). Pulse duration effect on the distribution of energetic particles produced by intense femtosecond laser pulses irradiating solids. Phys Plasmas 8, 3718-3723.

Zhidkov, A., Uesaka, M., Sasaki, A., \& Daido, H., (2002). Ion acceleration in a solitary wave by an intense picosecond laser pulse. Phys Rev Lett 89, 215002. 


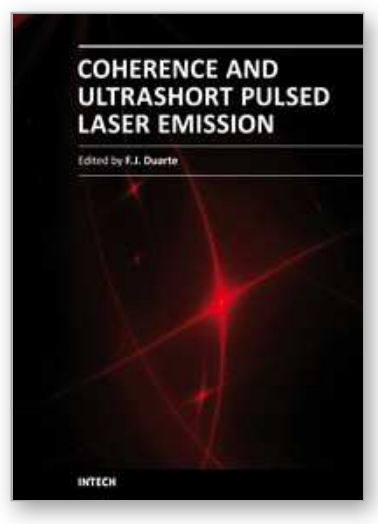

\section{Coherence and Ultrashort Pulse Laser Emission}

Edited by Dr. F. J. Duarte

ISBN 978-953-307-242-5

Hard cover, 688 pages

Publisher InTech

Published online 30, November, 2010

Published in print edition November, 2010

In this volume, recent contributions on coherence provide a useful perspective on the diversity of various coherent sources of emission and coherent related phenomena of current interest. These papers provide a preamble for a larger collection of contributions on ultrashort pulse laser generation and ultrashort pulse laser phenomena. Papers on ultrashort pulse phenomena include works on few cycle pulses, high-power generation, propagation in various media, to various applications of current interest. Undoubtedly, Coherence and Ultrashort Pulse Emission offers a rich and practical perspective on this rapidly evolving field.

\section{How to reference}

In order to correctly reference this scholarly work, feel free to copy and paste the following:

Sargis Ter-Avetisyan, Matthias Schnürer and Peter Victor Nickles (2010). Characterisation and Manipulation of Proton Beams Accelerated by Ultra-Short and High-Contrast Laser Pulses, Coherence and Ultrashort Pulse Laser Emission, Dr. F. J. Duarte (Ed.), ISBN: 978-953-307-242-5, InTech, Available from:

http://www.intechopen.com/books/coherence-and-ultrashort-pulse-laser-emission/characterisation-andmanipulation-of-proton-beams-accelerated-by-ultra-short-and-high-contrast-laser

\section{INTECH}

open science | open minds

\section{InTech Europe}

University Campus STeP Ri

Slavka Krautzeka 83/A

51000 Rijeka, Croatia

Phone: +385 (51) 770447

Fax: +385 (51) 686166

www.intechopen.com

\section{InTech China}

Unit 405, Office Block, Hotel Equatorial Shanghai

No.65, Yan An Road (West), Shanghai, 200040, China

中国上海市延安西路65号上海国际贵都大饭店办公楼405单元

Phone: +86-21-62489820

Fax: +86-21-62489821 
(C) 2010 The Author(s). Licensee IntechOpen. This chapter is distributed under the terms of the Creative Commons Attribution-NonCommercialShareAlike-3.0 License, which permits use, distribution and reproduction for non-commercial purposes, provided the original is properly cited and derivative works building on this content are distributed under the same license. 